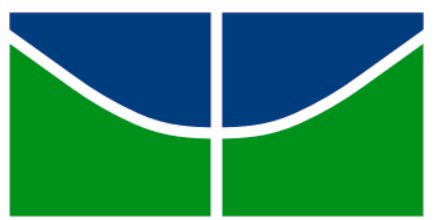

Universidade de Brasília

Instituto de Ciências Biológicas

Programa de Pós-Graduação em Zoologia

Diversidade de besouros rola-bosta (Coleoptera: Scarabaeidae: Scarabaeinae) em duas unidades de conservação do Cerrado do Brasil Central

MARCUS VINÍCIUS CELANI ROCHA 
Universidade de Brasília - UnB

Instituto de Ciências Biológicas

Departamento de Zoologia

Programa de Pós-Graduação em Zoologia

\title{
Diversidade de besouros rola-bosta (Coleoptera: Scarabaeidae: Scarabaeinae) em duas unidades de conservação do Cerrado do Brasil Central
}

\author{
MARCUS VINÍCIUS CELANI ROCHA
}

Dissertação de Mestrado apresentada ao Programa de Pós-Graduação em Zoologia do Instituto de Ciências Biológicas como requisito parcial para a obtenção do título de Mestre em Zoologia

Orientadora: Profa. Dra. Marina Regina Frizzas

Brasília - 2016 


\section{AGRADECIMENTOS}

Em primeiro lugar, gostaria de agradecer aos meus pais, Alfredo e Marlene, por todo apoio que me deram ao longo de toda minha vida, e por terem me proporcionado chegar à mais esta etapa, não medindo esforços para que eu alcançasse meus objetivos, principalmente no quesito educação.

Em seguida, gostaria de agradecer minha orientadora, Dra. Marina Frizzas. A Profa. Marina é uma pessoa extremamente paciente, e aguentou meus deslizes, atrasos, pisadas na bola, falação no laboratório... mas quando necessário, soube cobrar resultados, sem nunca faltar com respeito. Por isso, para mim, a Profa. Marina não é apenas minha orientadora, e sim minha amiga. Sem dúvida, sem a orientação que tive, o presente trabalho nunca existiria.

Ao Dr. Fernando Zagury Vaz-de-Mello (UFMT) pela identificação das espécies coletadas, essencial para os resultados do trabalho.

Ao Dr. Charles Martins de Oliveira (Embrapa Cerrados) pela ajuda com as análises estatísticas, meu calcanhar de Aquiles, e pelas fotos das espécies mais coletadas.

À minha grande amiga Juliane, que foi minha companheira de campo e de laboratório, inclusive aos sábados, acredita? E claro, também nas horas de descontração na mesa do bar.

Aos meus amigos de laboratório e campo, Túlio, sempre me ajudando em todas as coletas e triagem do material, Marcela e Maycon, pela ajuda no campo do PNB, Thiara e João pela ajuda no campo do PNCV. Sem vocês, esse trabalho não teria ido para a frente. Na verdade, não teria nem saído do papel.

Ao Programa de Pós-Graduação em Zoologia, e também à Universidade de Brasília (UnB), fornecendo não apenas o espaço físico, mas também um ensino de qualidade, com professores muito capacitados.

Ao Dr. Ricardo Bomfim Machado coordenador do projeto "Bases para a monitoração da biodiversidade do Cerrado do Brasil Central - Rede ComCerrado (PPBio/Geoma)" por viabilizar a execução do projeto do qual este trabalho faz parte.

À Coordenação de Aperfeiçoamento de Pessoal de Nível Superior (CAPES) pela concessão da bolsa de Mestrado. 


\section{SUMÁRIO}

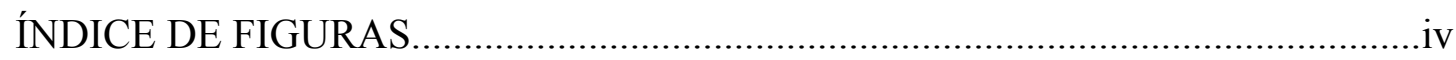

ÍNDICE DE TABELAS............................................................................................

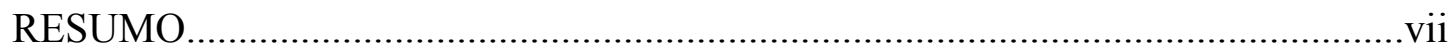

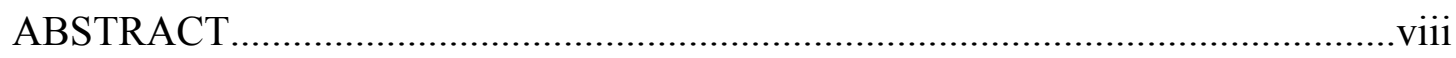

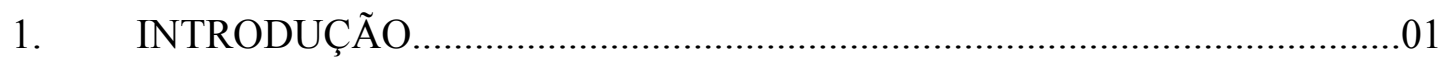

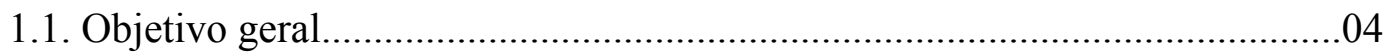

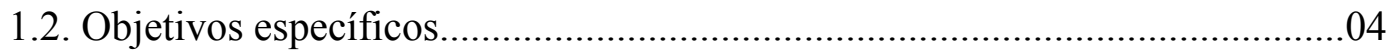

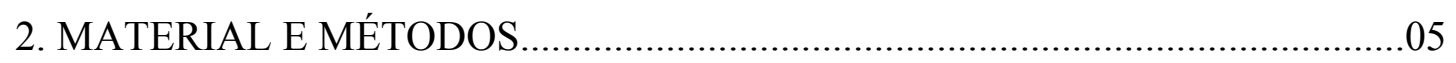

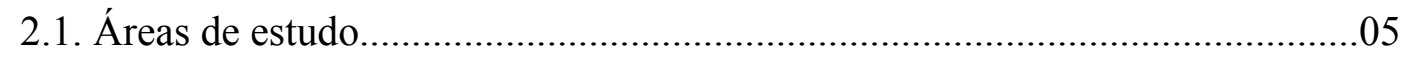

2.2. Coleta dos Scarabaeinae...................................................................................

2.3. Identificação dos Scarabaeinae coletados........................................................10

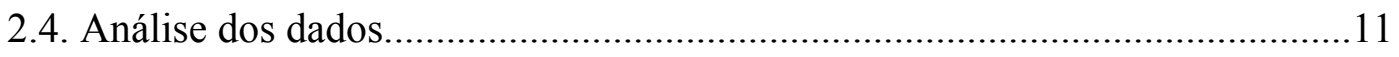

3. RESULTADOS

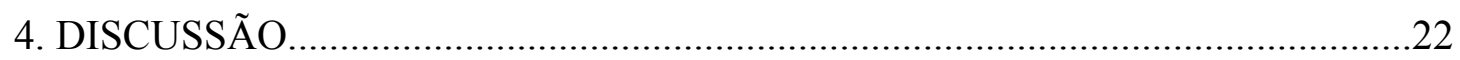

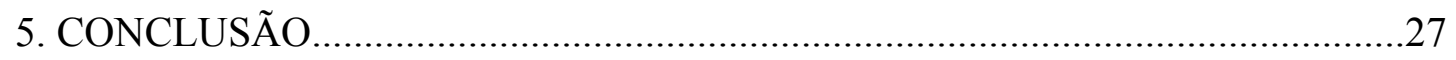

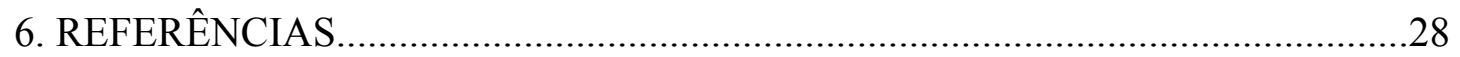




\section{ÍNDICE DE FIGURAS}

Figura 1. Perímetro do Parque Nacional de Brasília em vermelho. O retângulo vermelho no interior do parque representa o módulo de coleta dos Scarabaeinae

Figura 2. Perímetro do Parque Nacional da Chapada dos Veadeiros em vermelho. As duas linhas paralelas no interior do parque representam o módulo de coleta dos Scarabaeinae

Figura 3. A) Módulo de coleta de Scarabaeinae no Parque Nacional de Brasília, onde o Cerrado sensu strictu é predominante. B) Módulo de coleta de Scarabaeinae no Parque Nacional da Chapada dos Veadeiros, onde o Campo limpo é predominante .8

Figura 4. Esquema do módulo instalado nas duas unidades de conservação para a coleta dos Scarabaeinae. As linhas vermelhas paralelas representam os transectos norte e sul do módulo e as tachinhas amarelas, separadas $1 \mathrm{~km}$ entre si, representam as parcelas de coleta ao longo do transecto...... . .9

Figura 5. Armadilha tipo pitfall utilizada para coletar os Scarabaeinae no Parque Nacional de Brasília e no Parque Nacional da Chapada dos Veadeiros. 10

Figura 6. Espécies de Scarabaeinae mais abundantes no PNB. A) Oxysternon palemo B) Coprophanaeus spitzi C) Genieridium cryptops D) Canthidium decoratum. As linhas laterais representam uma escala de 10 milímetros. 16

Figura 7. Espécies de Scarabaeinae mais abundantes no PNCV. A) Dichotomius lycas B) Canthon aff. unicolor C) Uroxys sp. 1 D) Sulcophanaeus menelas. As linhas laterais representam uma escala de 10 milímetros. 16 
Figura 8. A) Curva de acumulação de espécies coletadas no PNB, utilizando como base o número de amostragens (pitfalls). B) Curva de acumulação de espécies coletadas no PNB, utilizando como base o número de indivíduos coletados.

Figura 9. A) Curva de acumulação de espécies coletadas no PNCV, utilizando como base o número de amostragens (pitfalls). B) Curva de acumulação de espécies coletadas no PNCV, utilizando como base o número de indivíduos coletados.

Figura 10. A) Valores médios de riqueza nas duas unidades de conservação avaliadas. B) Valores médios de abundância nas duas unidades de conservação avaliadas. As barras representam o desvio padrão e letras diferentes indicam diferenças significativas pelo teste $\mathrm{t}(\mathrm{p}<0,05)$ .20

Figura 11. Porcentagem relativa de guildas funcionais de Scarabaeinae para abundância e riqueza, no Parque Nacional de Brasília e no Parque Nacional da Chapada dos Veadeiros. 21

Figura 12. Porcentagem relativa do tamanho de Scarabaeinae para abundância e riqueza, no Parque Nacional de Brasília e no Parque Nacional da Chapada dos Veadeiros. 


\section{ÍNDICE DE TABELAS}

Tabela 1. Número de indivíduos por espécies e tribo de Scarabaeinae coletados com pitfalls iscados com fezes humanas entre dezembro de 2014 e dezembro de 2015 no Parque Nacional de Brasília/DF (PNB) e no Parque Nacional da Chapada dos Veadeiros/GO

(PNCV) .14 


\section{RESUMO}

Os besouros rola-bosta (Scarabaeoidea: Scarabaeidae: Scarabaeinae) estão distribuídos em diversos habitats, e utilizam vários recursos como fonte de alimento, principalmente fezes de mamíferos. Fezes são um recurso efêmero e energético, sendo assim altamente disputado pelos rola-bosta. Há poucos trabalhos com Scarabaeinae no Cerrado, e desta forma, as unidades de conservação amostradas neste trabalho não possuíam coleta prévia para o grupo em seus limites. Os objetivos deste trabalho foram avaliar a riqueza e abundância da fauna de rola-bostas em duas unidades de conservação; e comparar o tamanho corporal e as guildas funcionais das comunidades entre as unidades de conservação. O presente estudo foi realizado no Parque Nacional de Brasília/DF (PNB) e no Parque Nacional da Chapada dos Veadeiros/GO (PNCV). As coletas de Scarabaeinae ocorreram entre dezembro/2014 e dezembro/2015, em módulos de coleta, que eram compostos por dois transectos paralelos, com $5 \mathrm{~km}$ de extensão. Foram utilizados pitfalls iscados com fezes humanas, separados $50 \mathrm{~m}$ entre si, os quais permaneceram no campo por 48 horas. No PNB foram coletadas 38 espécies, sendo 17 exclusivas, Oxysternon palemo sendo a mais abundante (47,6\%), e 2.605 indivíduos. No PNCV foram coletadas 45 espécies, sendo 24 exclusivas, Dichotomius lycas sendo a mais abundante $(15,1 \%)$, e 1.021 indivíduos. A maioria das espécies de rola-bosta foi pequena, porém a maior abundância foi de besouros grandes. As espécies paracoprídeas dominaram as coletas nas duas unidades de conservação. Foi constatada diferença significativa na abundância dos Scarabaeinae coletados, porém para a riqueza não houve diferença significativa. De acordo com os resultados pode-se dizer que as duas unidades de conservação estão suportando a comunidade de Scarabaeinae em suas áreas.

Palavras chave: Besouros coprófagos, pitfall, Parque Nacional de Brasília, Parque Nacional da Chapada dos Veadeiros, comunidade, tamanho corporal, guildas funcionais. 


\begin{abstract}
Dung beetles (Scarabaeoidea: Scarabaeidae: Scarabaeinae) are distributed throughout several habitats, and they utilize different resources as food, such as mammal dung, mainly. Feces are an ephemeral and energetic resource, being highly disputed by dung beetles. There are only a few studies with Scarabaeinae in the Brazilian Cerrados, therefore, both conservation unities sampled in this study were never previously sampled for this group. The objectives were to sample the dung beetle fauna using richness and abundance in two conservation unities; and compare body size and functional guilds of the communities between the conservation unities. This study was carried out in Parque Nacional de Brasília/DF (PNB) and Parque Nacional da Chapada dos Veadeiros/GO (PNCV). Scarabaeinae sampling occurred from December/2014 to December/2015, in sampled areas, which were designed as two parallel transects of $5 \mathrm{~km}$ extension. Pitfall traps, separated $50 \mathrm{~m}$ from each other, and baited with human dung were installed. Each pitfall trap remained in the field for 48 hours. In PNB were sampled 2,605 individuals belonging to 38 species, 17 being exclusive, Oxysternon palemo being the most abundant (47,6\%). At PNCV, 1,021 individuals belonging to 45 species, 24 being exclusive, Dichotomius lycas being the most abundant $(15,1 \%)$. The majority of species were considered small, even though bigger beetles had higher abundance. In both conservation units, paracoprids dominated the samples. At PNB, species richness was inferior than PNCV, but the abundance was statistically higher. According to the results, both conservation unities are supporting the Scarabaeinae community in their limits.
\end{abstract}

Key words: Coprophagous beetles, pitfall, Parque Nacional de Brasília, Parque Nacional da Chapada dos Veadeiros, community, body size, functional guilds. 


\section{1 - INTRODUÇÃO}

Os besouros rola-bosta pertencem a superfamília Scarabaeoidea, família Scarabaeidae e subfamília Scarabaeinae. Os Scarabaeoidea são organismos encontrados em quase todos os biomas do planeta, representados por cerca de 20 mil espécies (Ronqui \& Lopes, 2006).

Os besouros da subfamília Scarabaeinae são comumente denominados rolabosta devido ao hábito de diversas espécies rolarem o recurso alimentar (normalmente fezes de mamíferos) para um determinado sítio próximo ao recurso (Vaz-de-Mello et al., 2011). Esta subfamília possui aproximadamente 7.000 espécies, distribuídas por diversos tipos de ambiente (Vaz-de-Mello et al., 2011), destas ao menos 713 espécies ocorrem no Brasil (MMA, 2016a), sendo no mínimo 323 exclusivas (Vaz-de-Mello, 2000).

Os Scarabaeinae utilizam várias fontes de alimento e possuem diversos hábitos alimentares, como frutos em decomposição (saprofagia), carcaças de animais (necrofagia), fungos (micofagia) e principalmente fezes de mamíferos (coprofagia) (Halffter \& Matthews, 1966; Filgueiras et al., 2009; Pessoa, 2010). Acredita-se que a evolução da dieta variada encontrada nos Scarabaeinae provém da extinção dos grandes mamíferos encontrados na região Neotropical que ocorreu no Pleistoceno, favorecendo a utilização de outros recursos além de fezes (Halffter, 1991; Filgueiras et al., 2009). Os rola-bosta podem ser divididos em três guildas funcionais, dependendo de como manipulam o recurso, sendo denominados telecoprídeos aqueles que formam bolas de fezes e as transportam, rolando-as normalmente com as tíbias do último par de pernas para longe da fonte de alimento, paracoprídeos aqueles que cavam túneis logo abaixo do recurso e endocoprídeos aqueles que vivem sobre, ou dentro do recurso, sem transportá-lo (Halffter \& Matthews, 1966; Zunino, 1991). Diferentemente de outros grupos, tal como os mamíferos, os rola-bosta adquirem os recursos da prole antes mesmo da postura dos ovos (Zunino, 1991), estratégia que visa aumentar a sobrevivência da mesma, já que a larva eclode em meio ao alimento e não se arrisca forrageando.

Fezes são um recurso altamente energético e extremamente disputado por insetos, por ser efêmero e distribuído de maneira desigual em um ambiente (Hanski, 1987). Normalmente os rola-bosta preferem fezes mais úmidas, pois os adultos se alimentam da fração líquida da excreta, e a formação de bolas é mais eficiente do que 
em fezes muito secas, pois a umidade deixa o recurso mais maleável, o que facilita a formação das bolas (Al-Houty \& Al-Musalam, 1997). O padrão do recurso também pode influenciar o consumo sendo que um padrão agregado é mais atrativo para os rola-bosta que um padrão disperso (Ponce-Santizo et al., 2006). Diversos trabalhos mostram que fezes humanas estão entre os recursos mais atrativos para os Scarabaeinae, podendo atrair um maior número de espécies (Larsen et al., 2008; Filgueiras et al., 2009; Sanchez, 2011; da Silva \& Di Mare, 2012; Da Silva et al., 2012), possivelmente pelo caráter onívoro da dieta humana.

Os hábitos alimentares dos rola-bosta os tornam ativos na ciclagem de nutrientes provenientes de matéria orgânica e no controle das populações de parasitas que ovipositam sobre fezes e carcaças (Nichols et al., 2008), como no caso de moscas, onde o tamanho relativo das taxas de mortalidade dos indivíduos pode ser afetado pela presença de besouros rola-bosta (Ridsdill-Smith et al., 1986), além de ajudar na aeração e fertilidade do solo, aumentar as taxas de mineralização do mesmo, e dispersar sementes (Vulinec, 2002; Alves et al., 2009; Amézquita \& Favila, 2010).

O tamanho corporal dos Scarabaeinae pode ser um fator importante para se inferir como uma espécie utiliza o recurso, ou o grau de degradação de uma área. No caso da utilização de recursos, os besouros de menor tamanho corporal são menos eficazes em enterrar o recurso no solo, geralmente sendo endocoprídeos (Koller et al., 2007). Em relação aos níveis de degradação do habitat, rola-bostas podem responder se a área se encontra mais preservada ou degradada de acordo com o tamanho médio das espécies da comunidade presente, onde os besouros de menor tamanho corporal dominam ambientes degradados, sendo os besouros grandes mais suscetíveis à impactos (Escobar et al., 2008; Gardner et al., 2008).

Comunidades neotropicais de rola-bosta sofrem cada vez mais com o aumento da ação antrópica e modificação de seus hábitats, bem como a fragmentação do mesmo (Nichols et al., 2007). Além do desmatamento, outro fator de impacto na redução das comunidades de rola-bosta é a caça de mamíferos, tendo em vista que este grupo produz o principal recurso alimentar para os Scarabaeinae (Andresen \& Laurance, 2007). Segundo Favila \& Halffter (1997), os escarabeíneos são muito sensíveis às mudanças na vegetação e na alteração da estrutura da comunidade de mamíferos de seu habitat. Há também trabalhos que sugerem a implementação de unidades de conservação em áreas de Cerrado através do monitoramento da comunidade de Scarabaeinae (Almeida \& Louzada, 2009), sendo que estes 
organismos podem ser utilizados como bioindicadores de maneira relativamente simples (Agoglitta et al., 2010). A comunidade de Scarabaeinae forma duas guildas distintas em ambientes de floresta tropical, uma em seu interior, e outra no exterior desmatado, com uma comunidade de transição no ecótono. Isto permite que mudanças na composição daquele ecossistema possam ser observadas pela comunidade de Scarabaeinae que se encontra na região (Favila \& Halffter, 1997).

Insetos são considerados bioindicadores eficazes devido à sua ampla distribuição, grande variedade de espécies, por serem facilmente amostrados (Favila \& Halffter, 1997), além da reconhecida importância ecológica e responderem à distúrbios de forma clara (Wink et al., 2005), podendo ser utilizados para medir o grau de impacto antrópico sobre o meio ambiente, de forma simplificada, ao invés de verificar o ecossistema como um todo (Rainio \& Niemela, 2003). Por estes motivos, diversos estudos foram realizados nos últimos anos utilizando insetos como bioindicadores, por exemplo: Carabidae (Rainio \& Niemela, 2003), Formicidae (Andersen et al., 2002), insetos edáficos (Wink et al., 2005) e os rola-bosta (McGeoch et al., 2002; Araújo et al., 2009; Agoglitta et al., 2010) sendo estes muito utilizados com esta finalidade.

Mesmo tendo em vista a importância do grupo em questão, ainda há limitações no conhecimento das espécies brasileiras de Scarabaeidae, e trabalhos de inventário podem sanar a falta de registros, ou até mesmo encontrar novas espécies (Vaz-de-Mello, 2000). Luçardo et al. (2014) demonstraram que em um intervalo de 30 anos (1982 até 2012) apenas 64 trabalhos sobre Scarabaeoidea foram realizados no Cerrado, e destes, somente 12 foram realizados em Goiás, e 7 no Distrito Federal.

Uma estratégia comum para proteger a biodiversidade é a priorização em proteger áreas ameaçadas que tenham uma rica diversidade e alto grau de endemismo (Lima \& Franco, 2014). Segundo Myers et al. (2000), áreas com alto grau de endemismo e rápida perda de habitat são classificadas como hotspots, e o Cerrado é uma das 25 áreas nesta categoria. Neste contexto, as unidades de conservação visam proteger áreas com estas características, lidando com a crescente ocupação humana. Segundo o site do Ministério do Meio Ambiente, unidades de conservação são áreas reservadas à preservação do patrimônio biológico, incluindo os recursos ambientais de características relevantes, com finalidade de manter amostras ecologicamente viáveis de populações, habitats e ecossistemas terrestres e aquáticos (MMA, 2016b).

O Ministério do Meio Ambiente classifica as unidades de conservação em dois 
grupos: (1) - Unidades de Proteção Integral, onde a proteção da biodiversidade é o foco principal, e o uso dos recursos naturais é feito de forma indireta, como ecoturismo, pesquisa científica, educação ambiental, dentre outras. As categorias de proteção integral são: estação ecológica, reserva biológica, parque, monumento natural e refúgio da vida silvestre; e (2) - Unidades de Uso Sustentável, onde a conservação se concilia ao uso sustentável dos recursos, sendo permitido o uso e coleta de recursos desde que os recursos renováveis sejam utilizados de forma adequada. São exemplos: área de relevante interesse ecológico, floresta nacional, reserva de fauna, reserva de desenvolvimento sustentável, reserva extrativista, área de proteção ambiental e reserva particular do patrimônio natural.

O Cerrado é um bioma bastante diverso, e muito de sua biodiversidade ainda está por ser descoberta. Ainda não há relatos de coletas de Scarabaeinae no Parque Nacional de Brasília (PNB), nem no Parque Nacional da Chapada dos Veadeiros, duas importantes unidades de conservação do Cerrado, caracterizadas como Unidades de Proteção Integral segundo o Ministério do Meio Ambiente. Como os rola-bosta são organismos sensíveis à impactos antrópicos, seu monitoramento dentro de unidades de conservação pode ser uma ferramenta útil para medir o grau de preservação dessas áreas, e verificar se estas estão funcionando de maneira eficiente para manter populações deste grupo, e consequentemente, de toda uma cadeia que deles depende.

O presente estudo foi vinculado ao Programa de Pesquisa em Biodiversidade (PPBio), que tem como finalidade incentivar trabalhos com a biodiversidade do nosso país, de forma descentralizada, integrando atividades de pesquisa e disponibilizando os resultados, para diversas finalidades. O PPBio está estruturado em três componentes, sendo eles: Coleções Biológicas, com suporte à coleções biológicas; Inventário Biológico, incentivando trabalhos de levantamento biológico; e Projetos Temáticos, que desenvolvem métodos de manejo sustentável (PPBio, 2012).

\section{1 - Objetivo geral}

Avaliar a fauna de besouros rola-bosta em duas unidades de conservação no Cerrado, verificando a riqueza e abundância das espécies.

\section{2 - Objetivos específicos}


A - Comparar as duas unidades de Conservação quanto ao tamanho corporal dos Scarabaeinae coletados.

B - Comparar as duas unidades de Conservação quanto à composição das guildas funcionais dos Scarabaeinae coletados.

\section{2 - MATERIAL E MÉTODOS}

2.1 - Áreas de estudo

Este estudo foi realizado no bioma Cerrado em duas unidades de conservação: Parque Nacional de Brasília (PNB) localizado em Brasília no Distrito Federal e Parque Nacional da Chapada dos Veadeiros (PNCV) localizado entre Cavalcante e Alto Paraíso de Goiás, em Goiás.

O Cerrado é o bioma da região central do Brasil, ocupando aproximadamente 2 milhões de $\mathrm{km}^{2}$, pouco mais de $20 \%$ do território nacional (Ratter et al., 1997; Klink \& Machado, 2005). Possui diversas fitofisionomias, desde as mais abertas como os campos, até as mais densas como o cerradão (Furley, 1998), além de um solo com baixo $\mathrm{pH}$, deficiente de nutrientes e saturado de alumínio (Mazorra et al., 1987; Ratter et al., 1997). Cerca de $47 \%$ do seu território ainda é composto por vegetação nativa (Beuchle et al., 2015), sendo que a parte norte do bioma é mais conservada que a parte sul (Sano et al., 2009).

O PNB (15 $42^{\prime} 53.92^{\prime} ' \mathrm{~S}, 47^{\circ} 59^{\prime} 30.58^{\prime}$ 'W e $1.200 \mathrm{~m}$ de altitude) se localiza à aproximadamente $10 \mathrm{~km}$ do centro de Brasília, na região noroeste do Distrito Federal (Figura 1). Foi criado em novembro de 1961 e atualmente possui pouco mais de 42 mil ha de área, abrigando bacias que fornecem cerca de $25 \%$ da água potável que abastece a capital. O PNB é composto por diversas fitofisionomias características do Cerrado, como Mata de galeria, Mata seca, Cerradão, Cerrado sensu strictu, Campo sujo, Campo limpo, Campo rupestre, Campo de murunduns, dentre outras. O clima da região é tropical, quente semi-úmido, com temperaturas médias anuais de $22^{\circ}$ a $24^{\circ} \mathrm{C}$. A umidade relativa fica abaixo dos $70 \%$ no período de maio a setembro, atingindo o mínimo de $47 \%$ no mês de agosto. O índice pluviométrico médio anual varia de 1.500 a $1.750 \mathrm{~mm}$ (ICMBio, 2016a). O parque possui uma zona de amortecimento bastante alterada, pois fica ao lado do aterro controlado da Estrutural, facilitando o acesso de 
pessoas e animais domésticos em sua área e, a caça, mesmo sendo estritamente proibida, é uma prática que ocorre nas suas dependências.

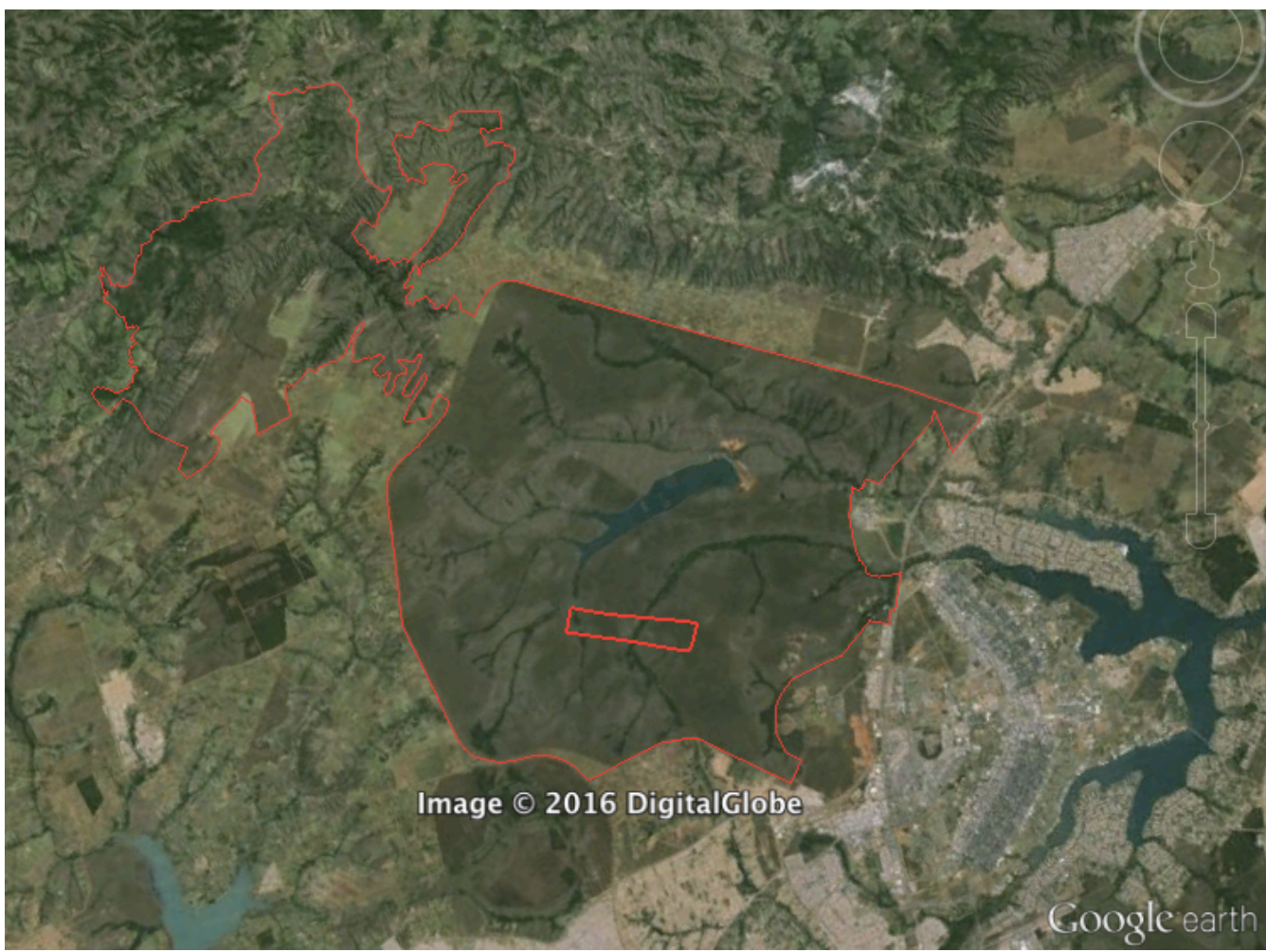

Figura 1. Perímetro do Parque Nacional de Brasília em vermelho. O retângulo vermelho no interior do parque representa o módulo de coleta dos Scarabaeinae.

O PNCV $\left(14^{\circ} 6^{\prime} 0.35^{\prime}\right.$ 'S, $47^{\circ} 41^{\prime} 55.55^{\prime \prime} \mathrm{W}$ e $1.500 \mathrm{~m}$ de altitude) se localiza na região nordeste do estado de Goiás, entre os municípios de Alto Paraíso de Goiás, Cavalcante e Colinas do Sul, à cerca de 260 km de Brasília (Figura 2). Possui cerca de 65 mil ha de Cerrado de altitude, e foi declarado como Patrimônio Natural da Humanidade pela UNESCO em 2001. O PNCV possui várias fitofisionomias, como: Campo rupestre, Campo limpo, Campo sujo, Cerrado sensu strictu, Cerradão, Mata de galeria, Vereda, dentre outras. O clima é tropical, quente semi-úmido, com temperaturas médias anuais entre $24^{\circ}$ a $26^{\circ} \mathrm{C}$. O índice pluviométrico médio anual vai de 1.500 a $1.750 \mathrm{~mm}$ (ICMBio, 2016b). O parque possui várias atrações turísticas, como trilhas, cachoeiras e cânions. No ano de 2014, cerca de 40 mil visitantes foram registrados em suas dependências. Apesar do ecoturismo, a região da Chapada dos 
Veadeiros é considerada bem conservada, possuindo o Parque Nacional da Chapada dos Veadeiros, APA do Pouso Alto, três parques municipais, sendo eles: PM Abílio Herculano Szervimsks, PM do Distrito de São Jorge e PM Lava-Pés, além de 15 Reservas Particulares do Patrimônio Natural (Lima \& Franco, 2014) nos seus arredores, aumentando a zona de amortecimento contra ações externas.

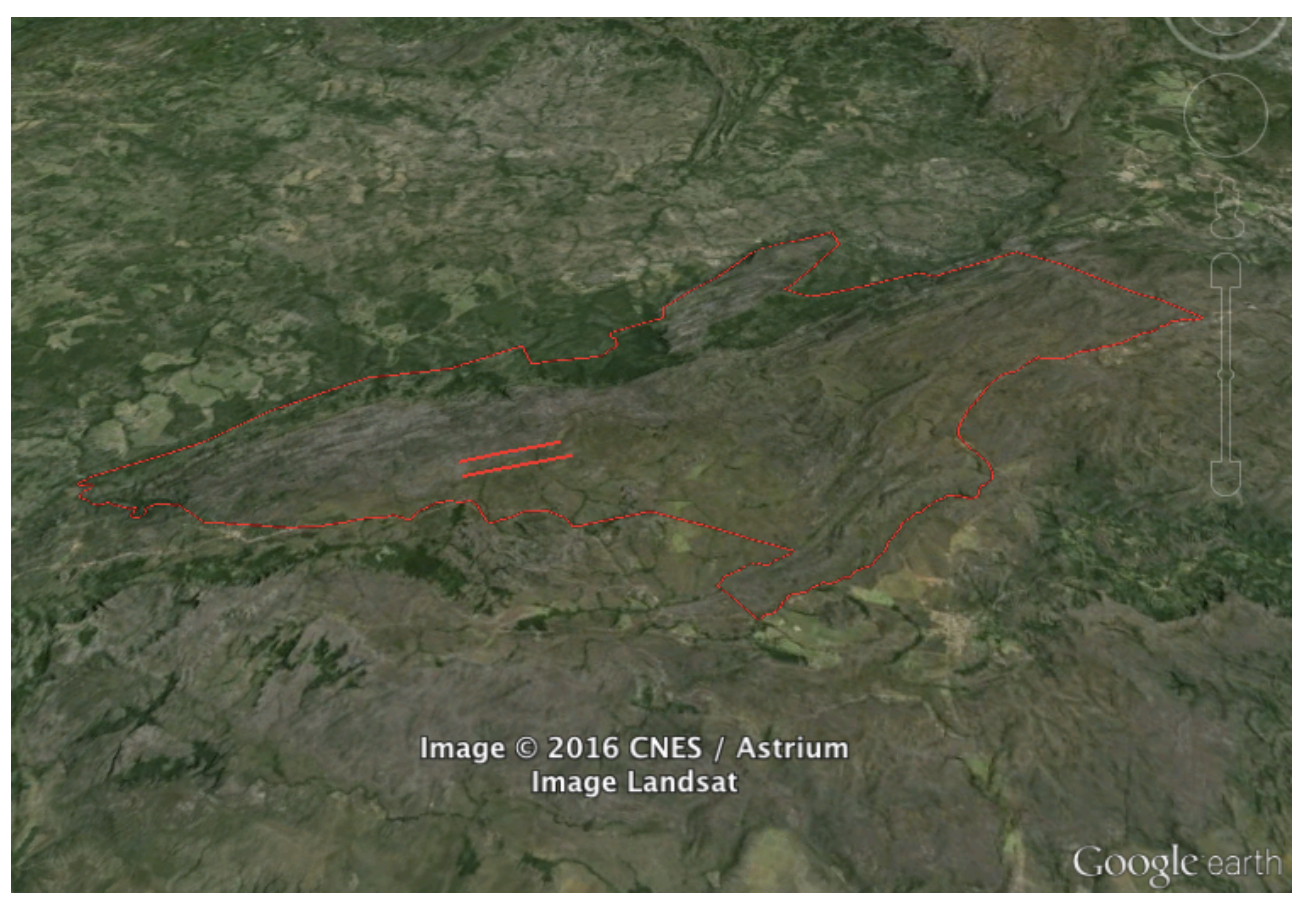

Figura 2. Perímetro do Parque Nacional da Chapada dos Veadeiros em vermelho. As duas linhas paralelas no interior do parque representam o módulo de coleta dos Scarabaeinae.

\section{2 - Coleta dos Scarabaeinae}

A coleta dos Scarabaeinae foi realizada em dois módulos pré-instalados. Os módulos foram instalados para a realização do projeto "Bases para a monitoração da biodiversidade do Cerrado do Brasil Central - Rede ComCerrado" aprovado no Edital MCTI/CNPq No. 35/2012 - PPBio/Geoma - Linha 5 - PPBio - Rede Cerrado com a finalidade de estudar diferentes grupos de organismos como por exemplo: plantas, aves, morcegos, pequenos mamíferos e insetos.

O módulo de coleta de Scarabaeinae no PNB era formado principalmente por áreas de Cerrado sensu stricto, ao passo que o módulo de coleta no PNCV era formado principalmente por áreas de Campo limpo (Figura 3). 

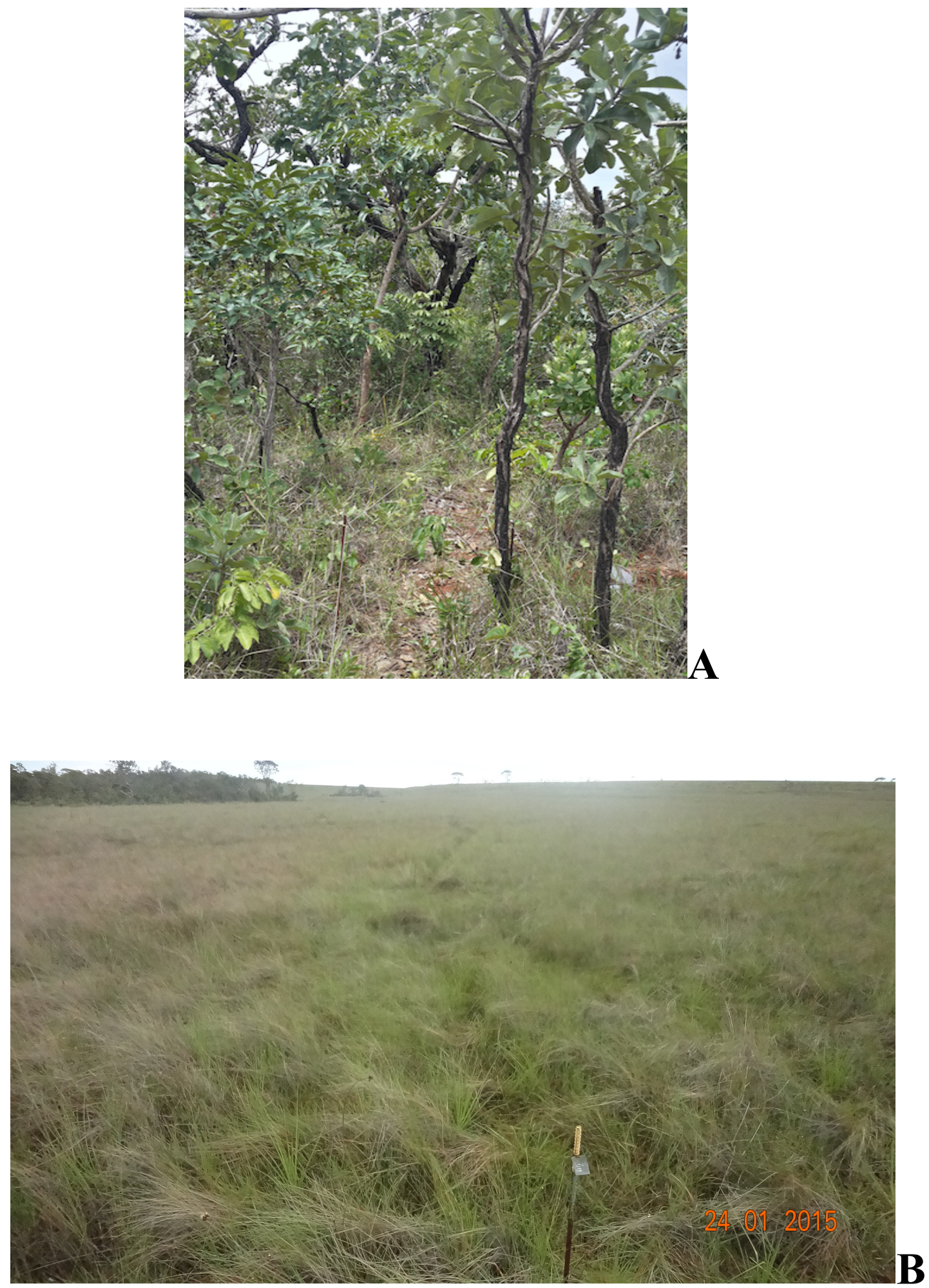

Figura 3. A) Módulo de coleta de Scarabaeinae no Parque Nacional de Brasília, onde o Cerrado sensu strictu é predominante. B) Módulo de coleta de Scarabaeinae no Parque Nacional da Chapada dos Veadeiros, onde o Campo limpo é predominante. 
Cada módulo era composto de dois transectos, um ao norte e outro ao sul, de 5 km de extensão, separados $1 \mathrm{~km}$ entre si. Para facilitar a localização no módulo, vergalhões com a metragem do local foram colocados por toda a extensão dos transectos a cada $50 \mathrm{~m}$. À cada quilômetro dos transectos, parcelas permanentes foram adicionadas, totalizando dez parcelas por módulo. A primeira parcela foi instalada à $500 \mathrm{~m}$ do início de um transecto, e a cada quilômetro subsequente, uma nova parcela foi instalada (Figura 4).

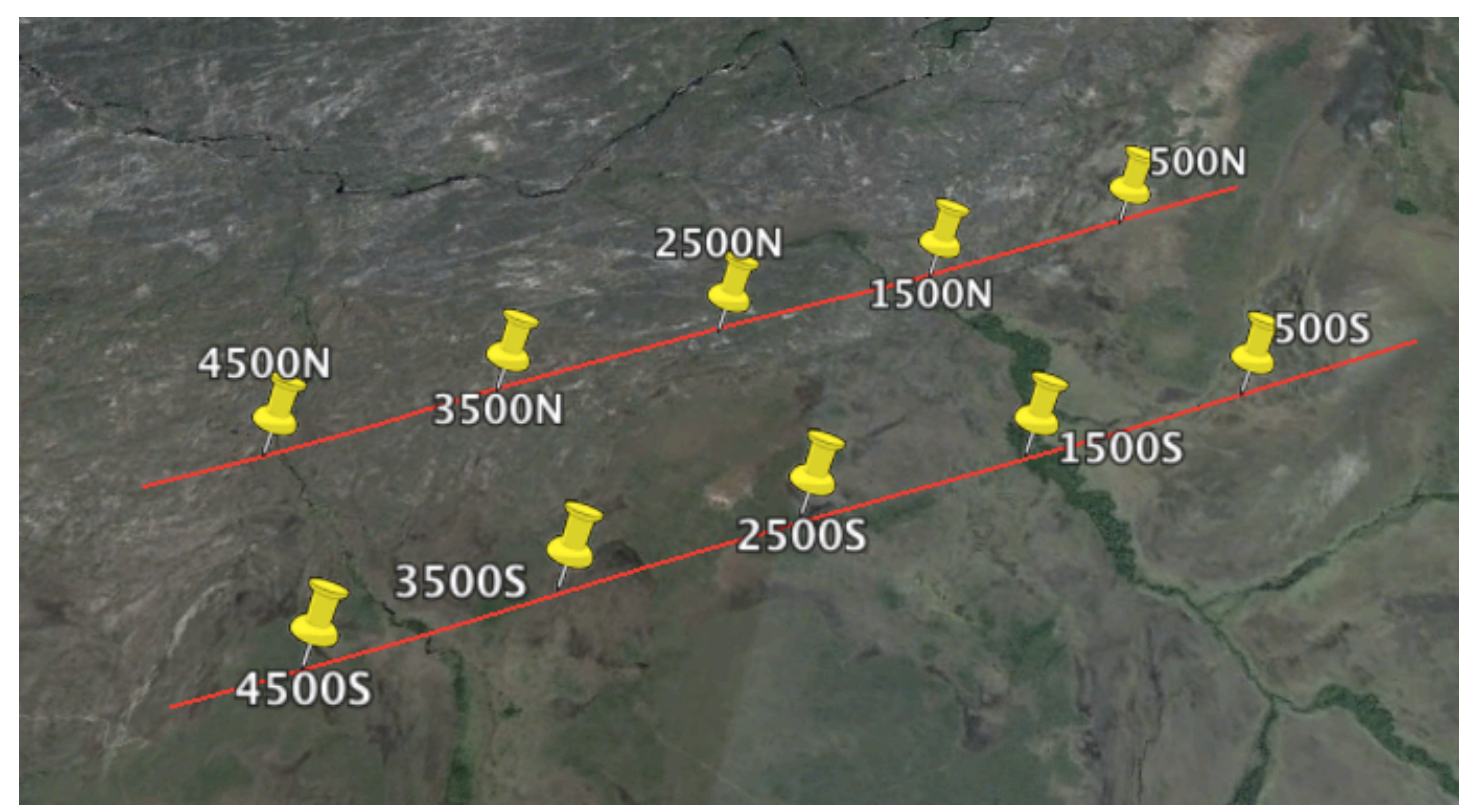

Figura 4. Esquema do módulo instalado nas duas unidades de conservação para a coleta dos Scarabaeinae. As linhas vermelhas paralelas representam os transectos norte e sul do módulo e as tachinhas amarelas, separadas $1 \mathrm{~km}$ entre si, representam as parcelas de coleta ao longo do transecto.

Foram realizadas três coletas no PNB no período compreendido entre dezembro de 2014 a março de 2015, e duas coletas no PNCV, uma em janeiro de 2015 e outra em dezembro do mesmo ano. Todas as coletas foram realizadas no período chuvoso, considerado a melhor época para a coleta de besouros rola-bosta (Santos \& Ávila, 2009; Lunz et al., 2011; Oliveira et al., 2011).

Os besouros foram coletados utilizando armadilhas do tipo pitfall iscadas com fezes humanas (Figura 5). A armadilha consistia de um recipiente cilíndrico plástico com volume de $1 \mathrm{~L}$ que era enterrado no nível do solo, contendo água e detergente. Em um recipiente menor, copo plástico de $80 \mathrm{ml}$, que ficava suspenso sobre o 
recipiente maior por meio de um pequeno fio de arame, era acondicionada a isca, cerca de $20 \mathrm{~g}$ de fezes humanas. Utilizando a tampa do recipiente maior e dois palitos para churrasco, um pequeno teto foi confeccionado a fim de proteger a armadilha da chuva.

Em cada parcela foram instalados 5 pitfalls distantes $50 \mathrm{~m}$ entre si e que permaneceram no campo por 48 horas. No PNB, foram instalados 45 pitfalls, distribuídos em 9 parcelas, e no PNCV foram instalados 30 pitfalls, distribuídos em 6 parcelas. As demais parcelas não foram amostradas devido ao solo ser extremamente duro e rochoso, não sendo possível cavar um local apropriado para a instalação dos pitfalls. Não houve pilhagem de armadilhas durante o período de coleta deste trabalho.

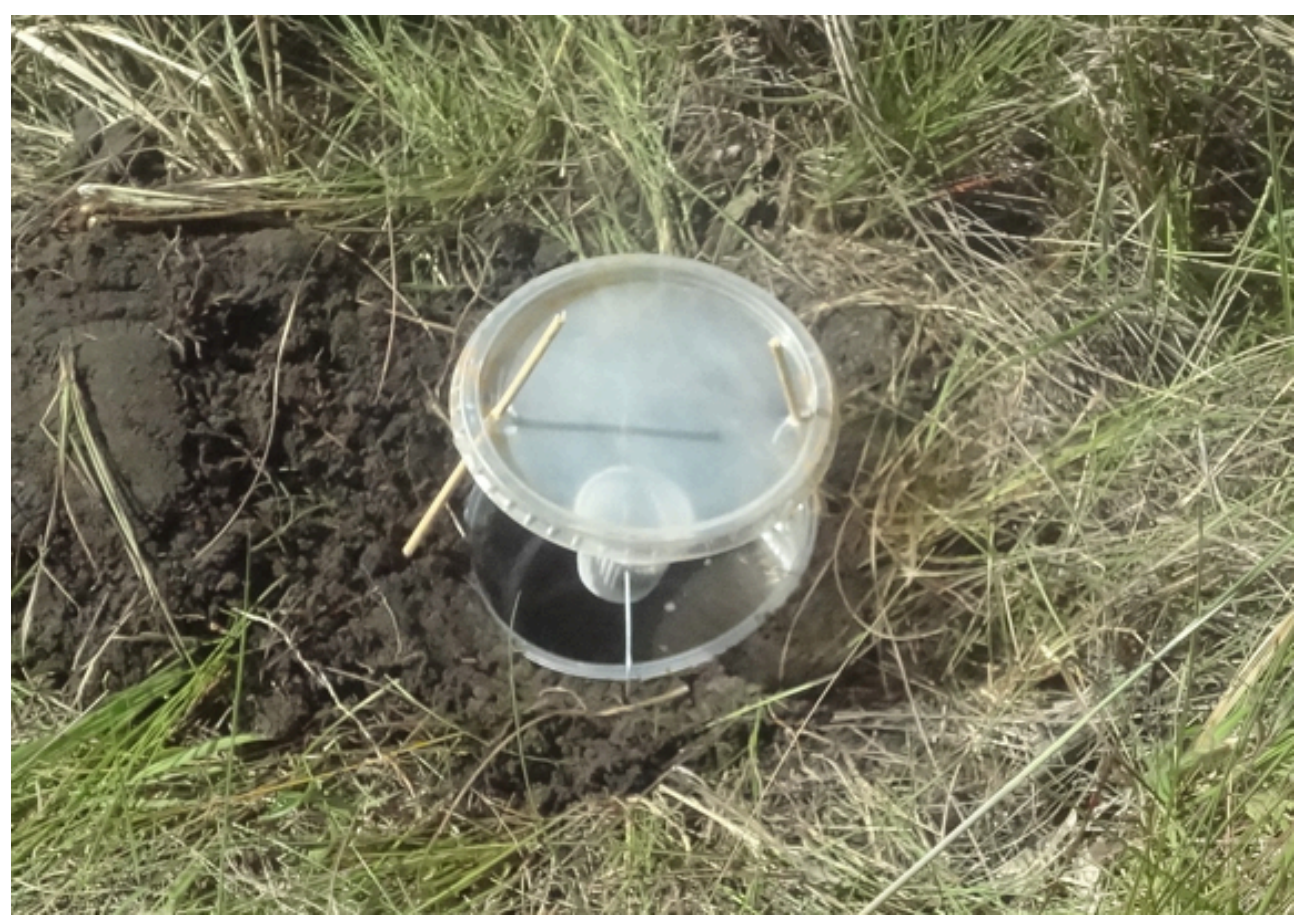

Figura 5. Armadilha tipo pitfall utilizada para coletar os Scarabaeinae no Parque Nacional de Brasília e no Parque Nacional da Chapada dos Veadeiros.

\section{3 - Identificação dos Scarabaeinae coletados}

Todos os Scarabaeinae coletados foram triados, morfoespeciados, montados, etiquetados e identificados em nível genérico utilizando chave de identificação (Vazde-Mello et al., 2011), bem como por comparação com indivíduos das coleções entomológicas da Universidade de Brasília e da Universidade Federal de Mato 
Grosso. A identificação em nível específico foi realizada com o auxílio do especialista Dr. Fernando Zagury Vaz-de-Mello (Departamento de Biologia e Zoologia do Instituto de Biociências da Universidade Federal de Mato Grosso, Cuiabá, MT). Os besouros coletados foram depositados na coleção entomológica do departamento de Zoologia da Universidade de Brasília e no Setor de Entomologia da Coleção Zoológica da Universidade Federal de Mato Grosso (CEMT).

\section{4 - Análise dos dados}

As espécies foram separadas em guildas funcionais (paracoprídeos, telecoprídeos e endocoprídeos) segundo Cambefort \& Hanski (1991) de acordo com os dados disponíveis na literatura. O comprimento do clípeo até o pigídeo foi medido, sempre que possível, para 10 indivíduos de cada espécie selecionados aleatoriamente, utilizando uma régua. As espécies foram divididas em grupos de acordo com o tamanho, conforme proposto por Escobar et al. (2008), em pequenas $(<10 \mathrm{~mm}) \mathrm{e}$ grandes $(>10 \mathrm{~mm})$. Para análise dos dados foram feitos gráficos de barras com as porcentagens relativas das duas variáveis (guilda funcional e tamanho).

Curvas de acumulação de espécies foram construídas com base no número de amostragens (pitfalls) e número de indivíduos. As curvas de acumulação de espécies, usando como base o índice de Jacknife 1 e 1.000 aleatorizações, foram obtidas utilizando-se o programa R version 3.2.3 (R Core Team, 2016).

A comparação da riqueza e abundância da comunidade de Scarabaeinae nas duas unidades de conservação foi feita pelo teste $t$ utilizando o programa R Versão 3.2.3 (R Core Team, 2016).

Para verificar a similaridade da comunidade de Scarabaeinae nas duas unidades de conservação, foi utilizado o índice de similaridade de Sorensen:

$$
S O=\frac{2 C}{A+B}
$$

Onde $\mathrm{C}$ representa o número de espécies comuns às duas unidades de conservação, A o número de espécies presentes no PNB e $\mathrm{B}$ o número de espécies presentes no PNCV.

Para verificar a diversidade de Scarabaeinae em cada unidade de conservação, utilizou-se o índice de Shannon-Wiener ( $\left.\mathrm{H}^{\prime}\right)$ : 


$$
\mathrm{H}^{\prime}=\frac{\left[\mathrm{N} \ln (\mathrm{N})-\sum_{i=1}^{S} n i \ln (n i)\right]}{N}
$$

Onde $\mathrm{N}$ é o número total de indivíduos, ni o número total de indivíduos da iésima espécie, $\mathrm{S}$ o número de espécies amostradas e ln o logaritmo de base neperiana. Os dois índices foram calculados por meio do programa Estimate $\mathrm{S}$ version 9.1 (Cowell, 2013).

\section{3 - RESULTADOS}

Foram coletados 3.626 indivíduos, separados em 25 gêneros, 62 espécies e seis tribos: Ateuchini (15 espécies e sete gêneros), Coprini (21 espécies e cinco gêneros), Deltochilini (10 espécies e seis gêneros), Eurysternini (duas espécies e um gênero), Onthophagini (três espécies e um gênero) e Phanaeini (11 espécies e cinco gêneros) (Tabela 1).

Considerando as duas unidades de conservação, as espécies mais abundantes foram Oxysternon palemo com 1.269 indivíduos (35\% do total), Coprophanaeus spitzi com 360 indivíduos (9,9\%), Genieridium cryptops com 199 indivíduos (5,5\%), Uroxys sp.1 com 180 indivíduos (5,0\%), Dichotomius lycas com 155 indivíduos (4,3\%), Canthon aff. unicolor com 154 indivíduos (4,2\%) e Uroxys sp.2 com 143 indivíduos (3,9\%). Estas sete espécies (11,3\% das espécies) representaram 67,8\% do total coletado.

No PNB foram coletados 2.605 indivíduos, os quais representaram $71,8 \%$ do total coletado, separados em 6 tribos, 20 gêneros e 38 espécies (Tabela 1). As espécies mais abundantes foram O. palemo (47,6\%), C. spitzi (13,8\%), G. cryptops (7,6\%) e Canthidium decoratum (4,4\%) (Figura 6) que juntas representaram $73,4 \%$ do total coletado. Já as espécies menos abundantes representadas por apenas um indivíduo cada $(0,04 \%)$ foram Agamopus viridis, Canthidium refulgens, Canthidium sp.3, Dichotomius bicuspis, Holocephalus julieni, Ontherus podiceps, Canthon lituratus e Deltochilum pseudoicarus.

Já no PNCV foram coletados 1.021 indivíduos (28,2\% do total coletado), separados em 6 tribos, 18 gêneros e 45 espécies (Tabela 1). As espécies mais abundantes foram D. lycas (15,1\%), C. aff. unicolor (14,6\%), Uroxys sp. 1 (13,3\%) e Sulcophanaeus menelas $(9,1 \%)$ (Figura 7) que representaram 52,1\% do total coletado. 
As espécies menos abundantes representadas por um indivíduo cada $(0,1 \%)$ foram Canthidium aff. luteum, C. refulgens, O. podiceps, Ontherus zikani, Canthonella sp. e C. spitzi.

Para cada unidade de conservação, duas curvas de acumulação de espécies foram construídas, uma usando como base as amostragens (pitfalls) e a outra o número de indivíduos (Figuras 8 e 9). Nenhuma das curvas se estabilizou, o que indica que mais armadilhas instaladas (no caso da curva por amostragem) e mais indivíduos capturados (no caso da curva por número de indivíduos) poderiam revelar outras espécies que não foram amostradas neste estudo. 
Tabela 1. Número de indivíduos por espécie e tribo de Scarabaeinae coletados com pitfalls iscados com fezes humanas entre dezembro de 2014 e dezembro de 2015 no Parque Nacional de Brasília/DF (PNB) e no Parque Nacional da Chapada dos Veadeiros/GO (PNCV).

\begin{tabular}{|c|c|c|c|c|}
\hline \multirow[b]{2}{*}{ Espécie } & \multicolumn{2}{|c|}{ Abundância } & \multirow[b]{2}{*}{ Tamanho ${ }^{1}$} & \multirow[b]{2}{*}{$\begin{array}{l}\text { Guilda } \\
\text { funcional }^{2}\end{array}$} \\
\hline & PNB & PNCV & & \\
\hline \multicolumn{5}{|l|}{ Ateuchini } \\
\hline Agamopus unguicularis (Harold) & - & 2 & $\mathrm{P}$ & $\mathrm{T}$ \\
\hline Agamopus viridis Boucomont & 1 & 3 & $\mathrm{P}$ & $\mathrm{T}$ \\
\hline Ateuchus aff. pygidialis (Harold) & - & 2 & $\mathrm{P}$ & $\mathrm{P}$ \\
\hline Ateuchus aff. viridemicans (Boucomont) & - & 8 & $\mathrm{P}$ & $\mathrm{P}$ \\
\hline Besourenga sp. & 20 & - & $\mathrm{P}$ & $\mathrm{E}$ \\
\hline Eutrichillum sp. & 2 & - & $\mathrm{P}$ & $\mathrm{E}$ \\
\hline Genieridium cryptops (Arrow) & 199 & - & $\mathrm{P}$ & $\mathrm{E}$ \\
\hline Trichillum adjunctum Martínez & 20 & - & $\mathrm{P}$ & $\mathrm{E}$ \\
\hline Trichillum arrowi Saylor & 2 & - & $\mathrm{P}$ & $\mathrm{E}$ \\
\hline Trichillum externepunctatum & - & 27 & $\mathrm{P}$ & $\mathrm{E}$ \\
\hline \multicolumn{5}{|l|}{ Preudhomme de Borre } \\
\hline Trichillum heydeni Harold & - & 18 & $\mathrm{P}$ & $\mathrm{E}$ \\
\hline Uroxys aff. epipleuralis (Boucomont) & 109 & 2 & $\mathrm{P}$ & $\mathrm{P}$ \\
\hline Uroxys sp. 1 & 44 & 136 & $\mathrm{P}$ & $\mathrm{P}$ \\
\hline Uroxys sp. 2 & 97 & 46 & $\mathrm{P}$ & $\mathrm{P}$ \\
\hline Uroxys sp. 3 & 5 & 19 & $\mathrm{P}$ & $\mathrm{P}$ \\
\hline \multicolumn{5}{|l|}{ Coprini } \\
\hline Canthidium aff. barbacenicum & 48 & 21 & $\mathrm{P}$ & $\mathrm{P}$ \\
\hline \multicolumn{5}{|l|}{ Preudhomme de Borre } \\
\hline Canthidium aff. luteum & - & 1 & $\mathrm{P}$ & $\mathrm{P}$ \\
\hline Canthidium aff. viride (Lucas) & 41 & - & $\mathrm{P}$ & $\mathrm{P}$ \\
\hline $\begin{array}{l}\text { Canthidium barbacenicum Preudhomme } \\
\text { de Borre }\end{array}$ & 34 & 50 & $\mathrm{P}$ & $\mathrm{P}$ \\
\hline Canthidium decoratum (Perty) & 116 & - & $\mathrm{P}$ & $\mathrm{P}$ \\
\hline Canthidium refulgens Boucomont & 1 & 1 & $\mathrm{P}$ & $\mathrm{P}$ \\
\hline Canthidium sp. 1 & 60 & 13 & $\mathrm{P}$ & $\mathrm{P}$ \\
\hline Canthidium sp. 2 & - & 3 & $\mathrm{P}$ & $\mathrm{P}$ \\
\hline Canthidium sp. 3 & 1 & - & $\mathrm{G}$ & $\mathrm{P}$ \\
\hline Canthidium sp. 4 & 4 & 11 & $\mathrm{P}$ & $\mathrm{P}$ \\
\hline $\begin{array}{l}\text { Chalcocopris inexpectatus Rossini \& } \\
\text { Vaz-de-Mello }\end{array}$ & 2 & - & G & $\mathrm{P}$ \\
\hline Dichotomius aff. fissiceps (Felsche) & - & 5 & $\mathrm{G}$ & $\mathrm{P}$ \\
\hline Dichotomius bicuspis (Germar) & 1 & - & $\mathrm{G}$ & $\mathrm{P}$ \\
\hline Dichotomius bos (Blanchard) & - & 5 & $\mathrm{G}$ & $\mathrm{P}$ \\
\hline Dichotomius lycas (Felsche) & - & 155 & $\mathrm{G}$ & $\mathrm{P}$ \\
\hline Dichotomius nisus (Oliver) & - & 2 & $\mathrm{G}$ & $\mathrm{P}$ \\
\hline Dichotomius zikani (Luederwaldt) & - & 24 & $\mathrm{G}$ & $\mathrm{P}$ \\
\hline Holocephalus julieni Smith \& Génier & 1 & - & $\mathrm{G}$ & $P$ \\
\hline
\end{tabular}




\begin{tabular}{|c|c|c|c|c|}
\hline \multirow[b]{2}{*}{ Espécie } & \multicolumn{2}{|c|}{ Abundância } & \multirow[b]{2}{*}{ Tamanho $^{1}$} & \multirow[b]{2}{*}{$\begin{array}{c}\text { Guilda } \\
\text { funcional }\end{array}$} \\
\hline & PNB & PNCV & & \\
\hline Ontherus appendiculatus (Mannerheim) & - & 5 & $\mathrm{G}$ & $\mathrm{P}$ \\
\hline Ontherus podiceps Harold & 1 & 1 & $\mathrm{G}$ & $\mathrm{P}$ \\
\hline Ontherus zikani Luederwaldt & - & 1 & $\mathrm{G}$ & $\mathrm{P}$ \\
\hline \multicolumn{5}{|l|}{ Deltochilini } \\
\hline Anisocanthon sp. & - & 4 & $\mathrm{P}$ & $\mathrm{T}$ \\
\hline Anomiopus pereirai Martínez & 2 & - & $\mathrm{P}$ & ND \\
\hline Canthon aff. pilluliformis Blanchard & - & 24 & $\mathrm{P}$ & $\mathrm{T}$ \\
\hline Canthon aff. unicolor Blanchard & 5 & 149 & $\mathrm{G}$ & $\mathrm{T}$ \\
\hline Canthon lituratus Germar & 1 & - & $\mathrm{P}$ & $\mathrm{T}$ \\
\hline Canthonella sp. & - & 1 & $\mathrm{P}$ & $\mathrm{T}$ \\
\hline Deltochilum aff. komareki Balthasar & 63 & 36 & $\mathrm{G}$ & $\mathrm{T}$ \\
\hline Deltochilum elevatum (Castelnau) & - & 3 & G & $\mathrm{T}$ \\
\hline Deltochilum pseudoicarus (Balthasar) & 1 & 21 & $\mathrm{G}$ & $\mathrm{T}$ \\
\hline Vulcanocanthon sp. & - & 3 & $\mathrm{P}$ & $\mathrm{T}$ \\
\hline \multicolumn{5}{|l|}{ Eurysternini } \\
\hline Eurysternus caribaeus (Herbst) & 5 & 6 & G & $\mathrm{E}$ \\
\hline Eurysternus nigrovirens Génier & 6 & 5 & $\mathrm{P}$ & $\mathrm{E}$ \\
\hline \multicolumn{5}{|l|}{ Onthophagini } \\
\hline Onthophagus buculus Mannerheim & 4 & 7 & $\mathrm{P}$ & $\mathrm{T}$ \\
\hline Onthophagus ptox Erichson & - & 4 & $\mathrm{P}$ & $\mathrm{T}$ \\
\hline Onthophagus sp. & 22 & 57 & $\mathrm{P}$ & $\mathrm{T}$ \\
\hline \multicolumn{5}{|l|}{ Phanaeini } \\
\hline Coprophanaeus acrisius (MacLeay) & - & 3 & $\mathrm{G}$ & $\mathrm{P}$ \\
\hline Coprophanaeus ensifer (Germar) & 21 & - & $\mathrm{G}$ & $\mathrm{P}$ \\
\hline Coprophanaeus horus (Waterhouse) & 9 & - & $\mathrm{G}$ & $\mathrm{P}$ \\
\hline Coprophanaeus spitzi (Pessôa) & 359 & 1 & $\mathrm{G}$ & $\mathrm{P}$ \\
\hline Diabroctis mirabilis (Harold) & 26 & - & G & $\mathrm{P}$ \\
\hline Oxysternon palemo Castelnau & 1240 & 29 & $\mathrm{G}$ & $\mathrm{P}$ \\
\hline Phanaeus bispinus Bates & - & 2 & G & $\mathrm{P}$ \\
\hline Phanaeus kirbyi Vigors & 5 & - & $\mathrm{G}$ & $\mathrm{P}$ \\
\hline Phanaeus melibaeus Blanchard & - & 10 & $\mathrm{G}$ & $\mathrm{P}$ \\
\hline Phanaeus palaeno Blanchard & 27 & 2 & $\mathrm{G}$ & $\mathrm{P}$ \\
\hline Sulcophanaeus menelas (Castelnau) & - & 93 & $\mathrm{G}$ & $\mathrm{P}$ \\
\hline Total & 2.605 & 1.021 & & \\
\hline
\end{tabular}

*1. $\mathrm{P}=$ Pequeno e $\mathrm{G}=$ Grande. 2. $\mathrm{P}=$ Paracoprídeo, $\mathrm{T}=$ Telecoprídeo, $\mathrm{E}=$ Endocoprídeo e ND = Não Determinado. 
A)

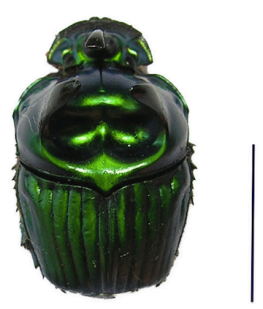

C)

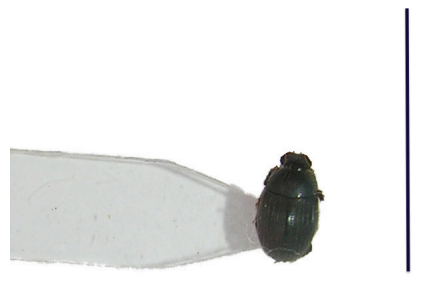

B)

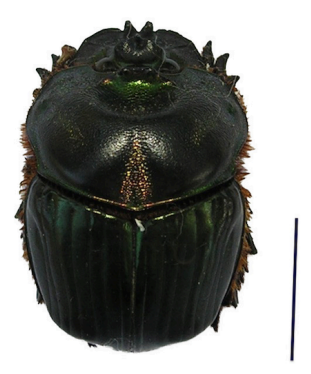

D)

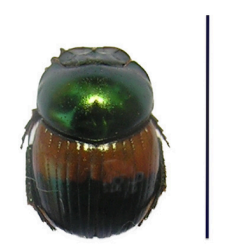

Figura 6. Espécies de Scarabaeinae mais abundantes no PNB. A) Oxysternon palemo B) Coprophanaeus spitzi C) Genieridium cryptops D) Canthidium decoratum. As linhas laterais representam uma escala de 10 milímetros.

A)

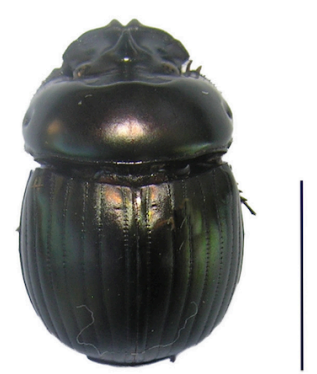

C)

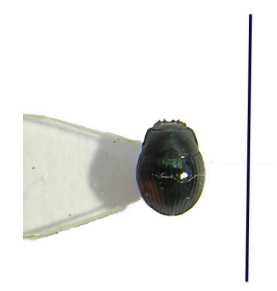

B)

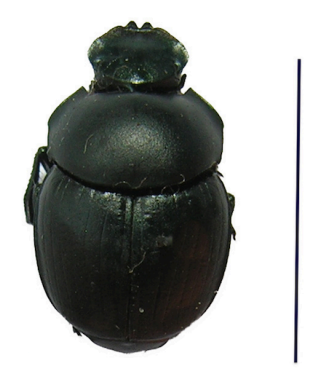

D)

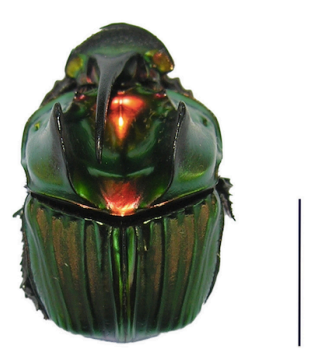

Figura 7. Espécies de Scarabaeinae mais abundantes no PNCV. A) Dichotomius lycas B) Canthon aff. unicolor C) Uroxys sp. 1 D) Sulcophanaeus menelas As linhas laterais representam uma escala de 10 milímetros. 

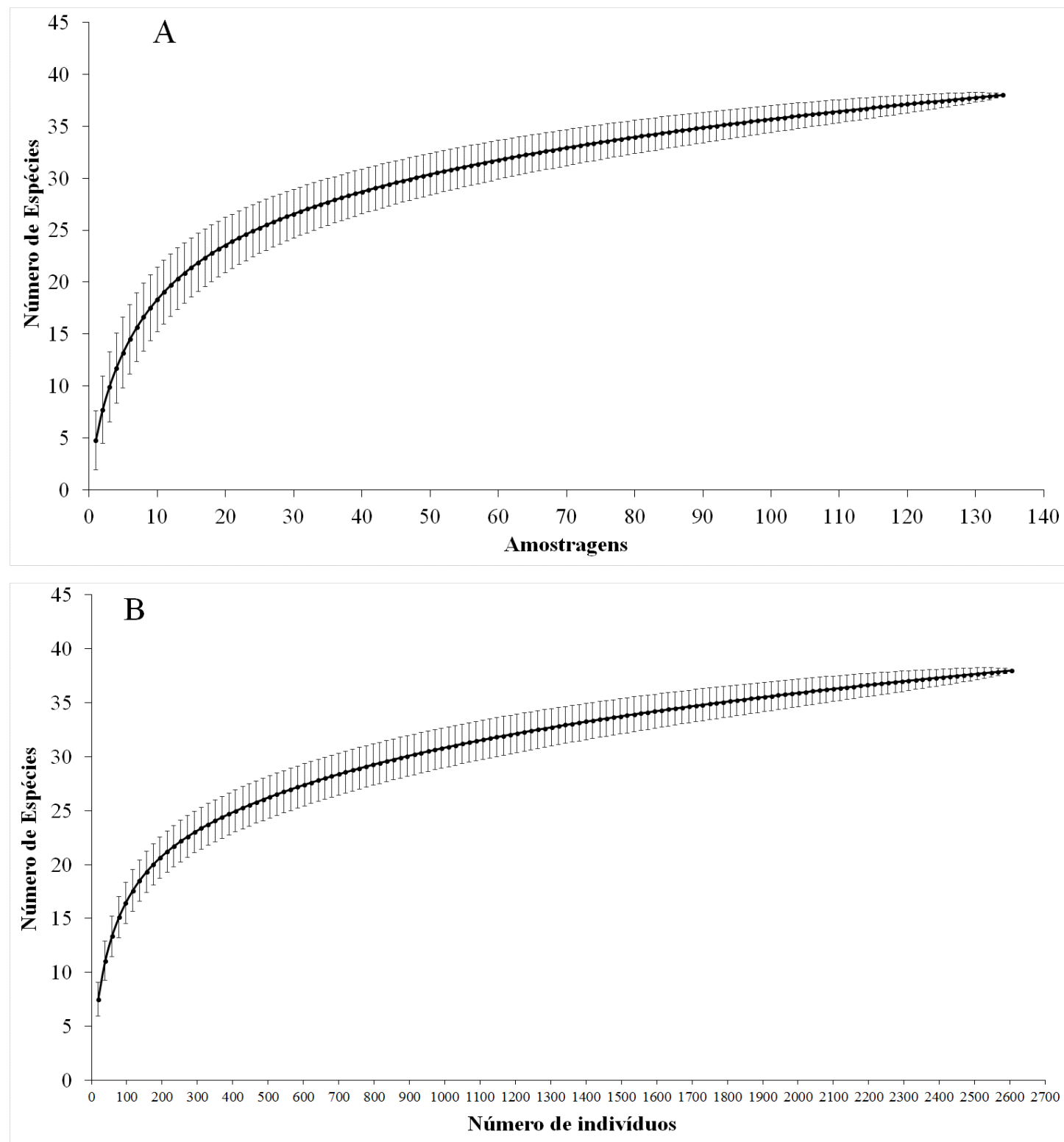

Figura 8. A) Curva de acumulação de espécies coletadas no PNB, utilizando como base o número de amostragens (pitfalls). B) Curva de acumulação de espécies coletadas no PNB, utilizando como base o número de indivíduos coletados. 

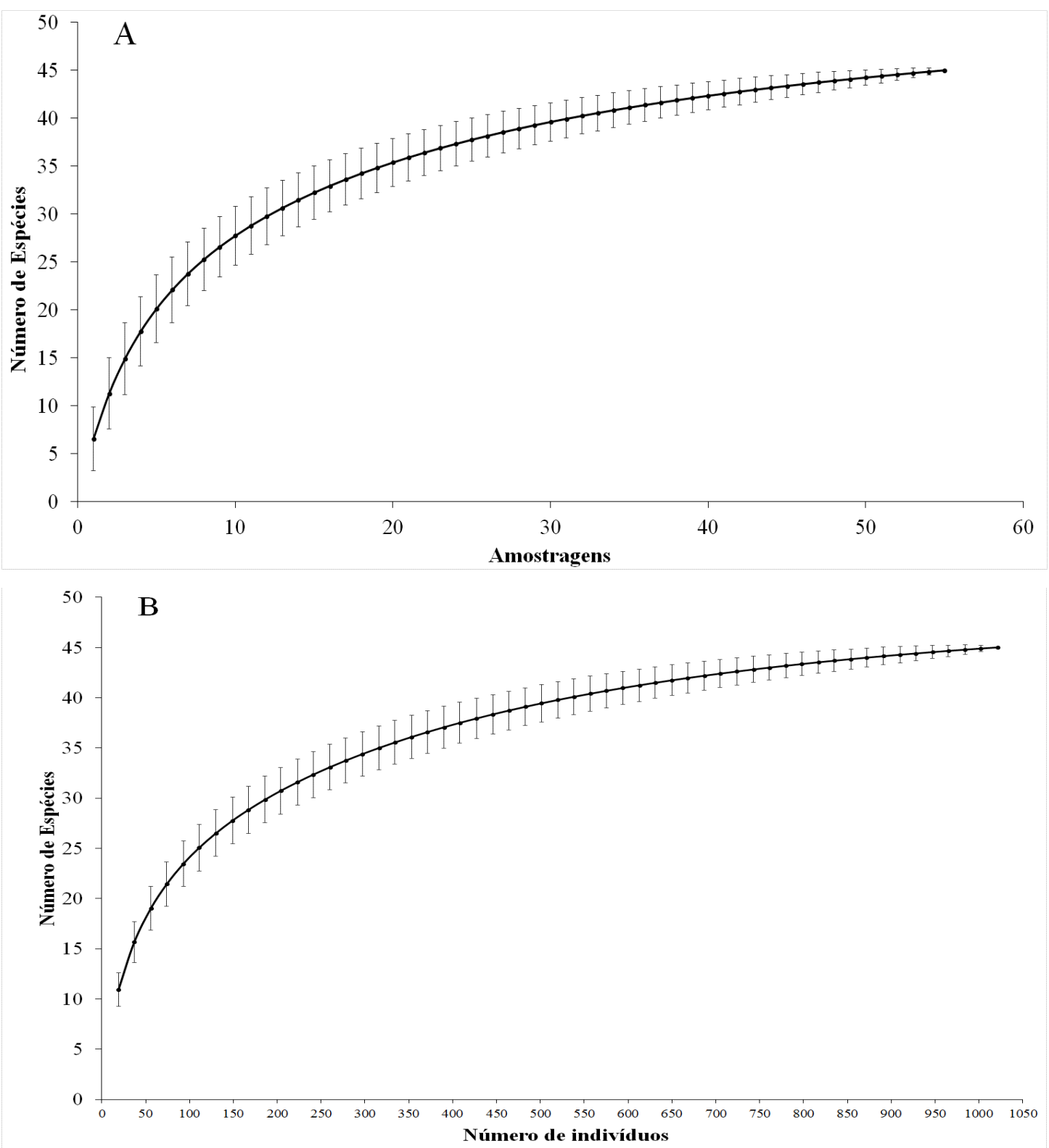

Figura 9. A) Curva de acumulação de espécies coletadas no PNCV, utilizando como base o número de amostragens (pitfalls). B) Curva de acumulação de espécies coletadas no PNCV, utilizando como base o número de indivíduos coletados.

Das 62 espécies coletadas, 17 ocorreram apenas no PNB, e 24 apenas no PNCV, sendo as demais (23 espécies - 37,1\%) encontradas nas duas unidades de conservação (Tabela 1). Das exclusivas do PNB, as mais abundantes foram $G$. cryptops, C. decoratum, Canthidium aff. viride, Diabroctis mirabilis e Coprophanaeus ensifer. No PNCV, as principais espécies exclusivas foram D. lycas, $S$ menelas, Trichillum externepunctatum, Canthon aff. pilluliformis e D. zikani. A 
espécie Anisocanthon sp. é uma nova espécie para o gênero, e foi coletada apenas no PNCV.

O PNCV possui maior riqueza de espécies comparado ao PNB, porém o PNB apresentou maior abundância. De acordo com o teste t, não houve diferença significativa para a riqueza de espécies entre as unidades de conservação avaliadas ( $t$ $=-0,3320, \mathrm{gl}=46, \mathrm{p}=0,7413)$ mas houve diferença significativa para o número de indivíduos coletados $(\mathrm{t}=3,7525, \mathrm{gl}=52, \mathrm{p}=0,0004)$ (Figura 10).

Os valores do índice de diversidade de Shannon-Wiener (H') foram 2,898 no PNCV e 2,055 no PNB. O índice de similaridade de Sorensen foi de 46,08\% demonstrando que as unidades de conservação avaliadas possuem comunidades distintas de rola-bosta.

Espécies representativas das três guildas funcionais foram coletadas nas duas unidades de conservação (Tabela 1). Nas duas unidades de conservação, houve maior número de espécies paracoprídeas $(61,3 \%, \mathrm{n}=38)$, seguidas das telecoprídeas $(22,6 \%, \mathrm{n}=14)$ e endocoprídeas $(14,5 \%, \mathrm{n}=9)$, com uma espécie indeterminada (1,6\%). No PNB, as espécies paracoprídeas representaram $60,5 \%$ do total, seguido pelos telecoprídeos 18,4\% e endocoprídeos 18,4\%, com uma espécie de guilda indeterminada (2,6\%). No PNCV a guilda dos paracoprídeos representou 62,2\% das espécies coletadas, seguida pelos telecoprídeos $28,9 \%$ e endocoprídeos $8,9 \%$ (Figura 11).

Foram coletadas 34 espécies pequenas $(54,8 \%)$ e 28 grandes (45,2\%) (Figura 12). No PNB, coletou-se 22 espécies pequenas (57,9\%), sendo as 16 espécies restantes grandes $(42,1 \%)$. Das 22 espécies pequenas coletadas, 16 são paracoprídeas (72,7\%), e das 16 espécies grandes, 12 são paracoprídeas (75\%). No PNCV, foram capturadas 25 espécies pequenas (55,5\%) e 20 grandes (44,4\%). Das 25 espécies pequenas, 21 são paracoprídeas (84\%), e das 20 grandes, 15 são paracoprídeas (75\%).

No presente trabalho, a guilda funcional dos paracoprídeos apresentou riqueza e abundância mais expressiva nas duas unidades de conservação. No PNB, 28 espécies foram paracoprídeas $(73,6 \%$ do total), sendo a maioria dessas espécies pequenas $(57,1 \%)$. A abundância de paracoprídeos grandes foi muito superior a qualquer outra combinação de guilda funcional e tamanho $(65 \% \mathrm{n}=1.693)$, seguido pelos paracoprídeos pequenos $(21,4 \% \mathrm{n}=559)$. No PNCV, 36 espécies foram paracoprídeas (80\%), sendo a maioria pequena $(58,3 \%)$. A abundância de 
paracoprídeos pequenos e grandes foi semelhante, sendo os pequenos representados por $30,6 \%$ e $n=313$ e os grandes por $33,1 \%$ e $n=338$.
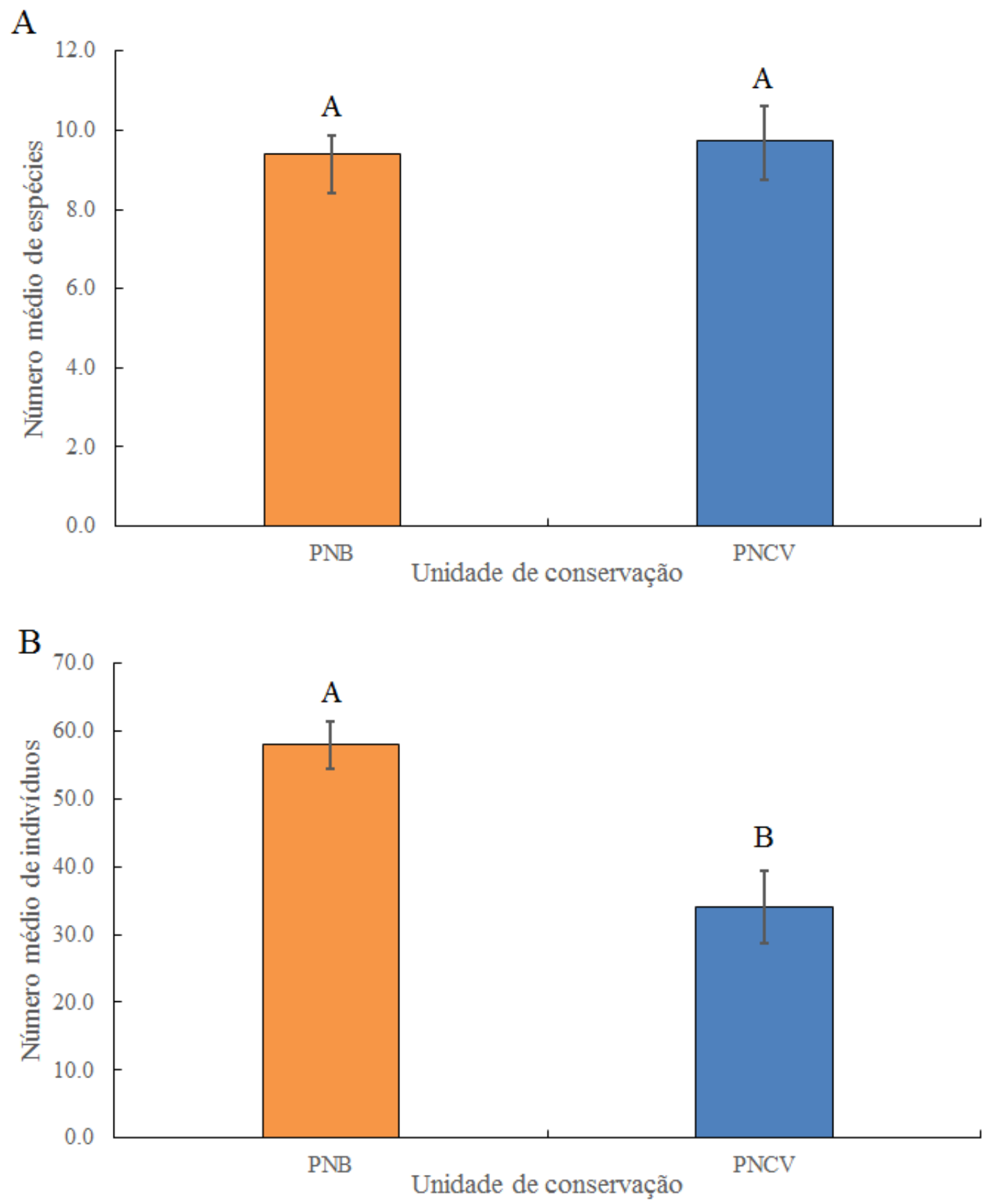

Figura 10. A) Valores médios de riqueza nas duas unidades de conservação avaliadas. B) Valores médios de abundância nas duas unidades de conservação avaliadas. As barras representam o desvio padrão e letras diferentes indicam diferenças significativas pelo teste $\mathrm{t}(\mathrm{p}<0,05)$. 


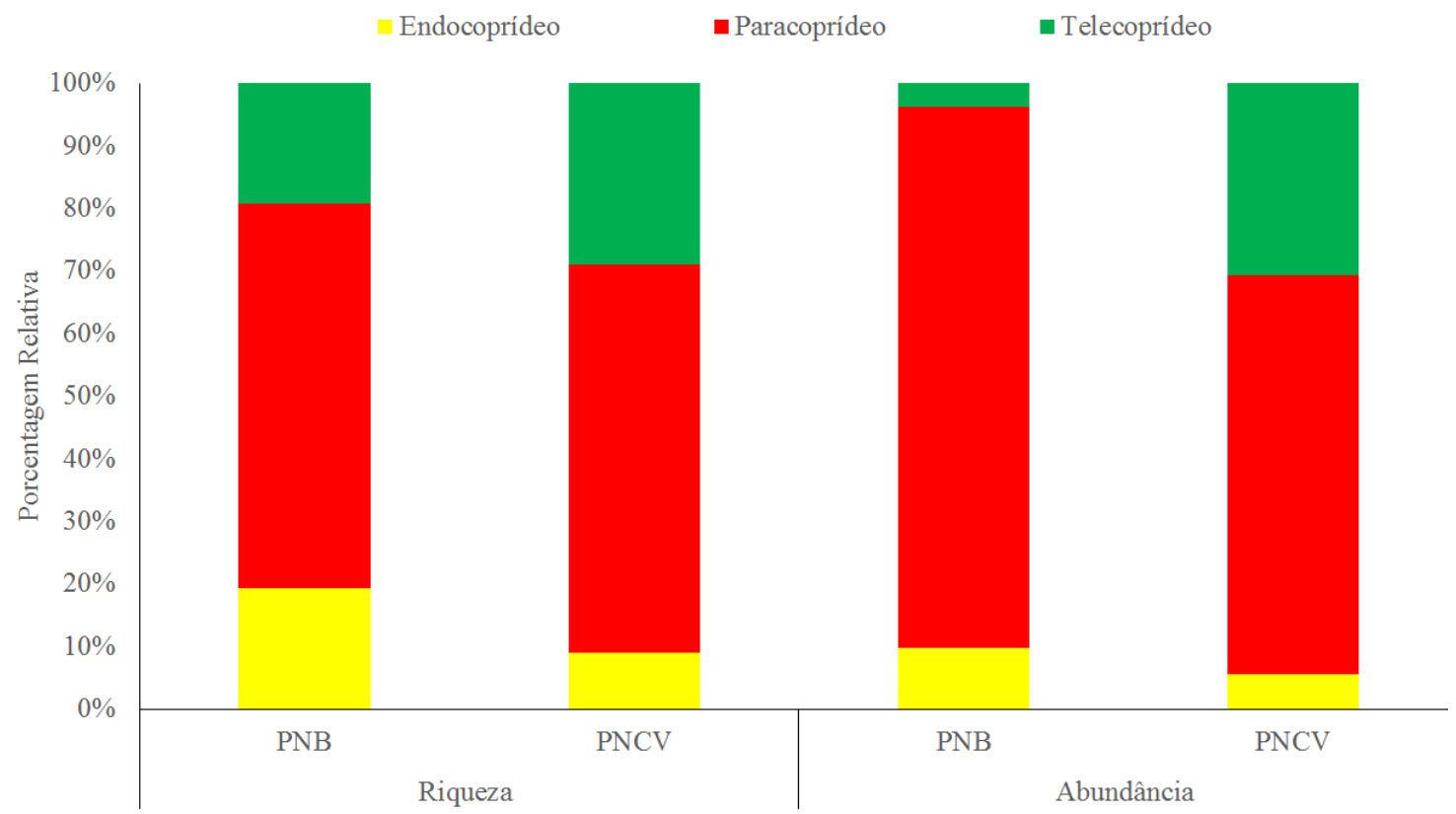

Figura 11. Porcentagem relativa de guildas funcionais de Scarabaeinae para abundância e riqueza, no Parque Nacional de Brasília (PNB) e no Parque Nacional da Chapada dos Veadeiros (PNCV).

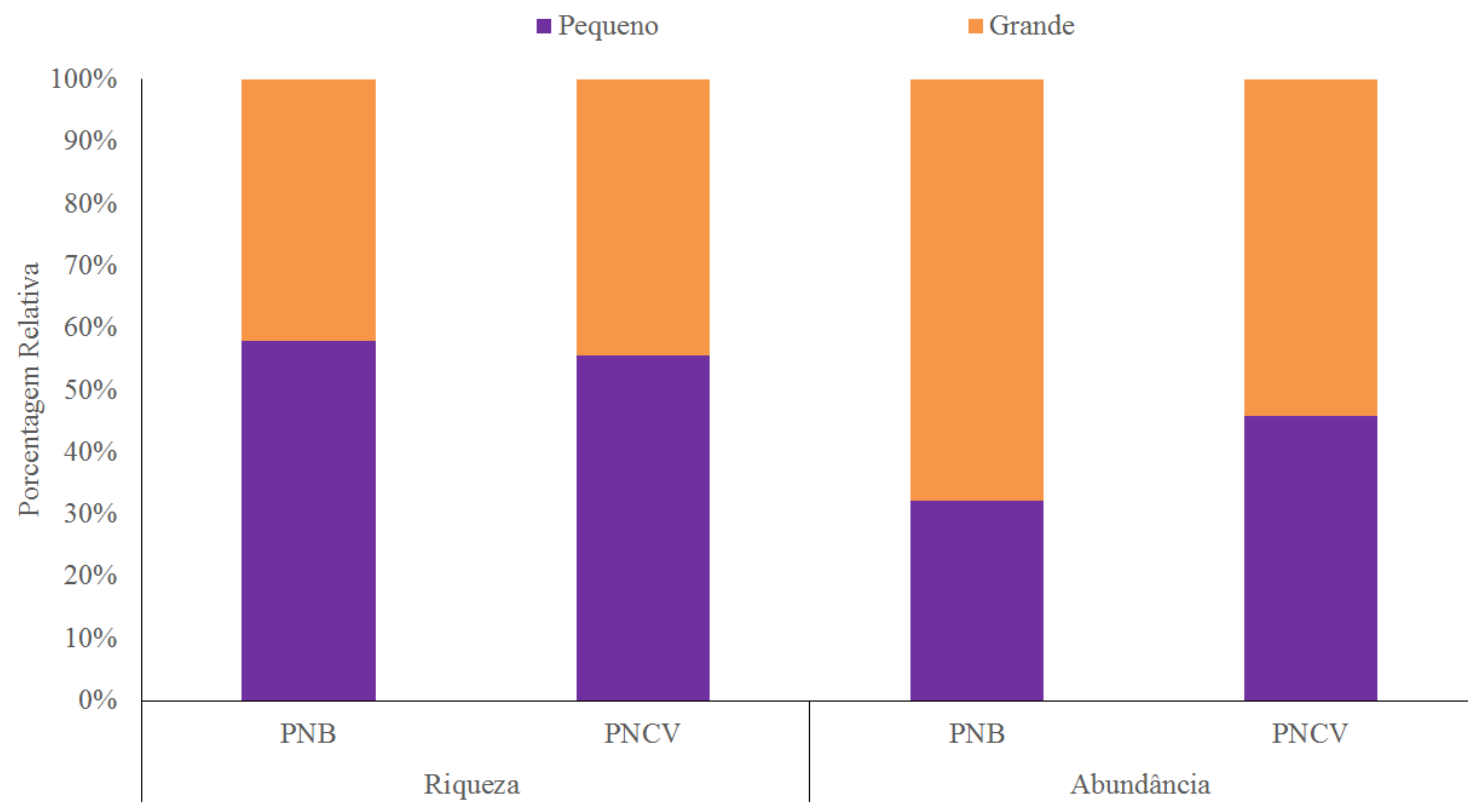

Figura 12. Porcentagem relativa do tamanho de Scarabaeinae para abundância e riqueza, no Parque Nacional de Brasília (PNB) e no Parque Nacional da Chapada dos Veadeiros (PNCV). 


\section{4 - DISCUSSÃO}

No presente trabalho, foram coletadas 62 espécies de besouros rola-bosta em duas importantes unidades de conservação do bioma Cerrado, sendo apenas 23 espécies comuns aos dois parques. Foi constatada diferença significativa na abundância dos Scarabaeinae coletados no PNB ( $\mathrm{n}=2.605$ indivíduos) e no PNCV (n $=1.021$ indivíduos), porém para a riqueza não houve diferença significativa, apesar de o PNCV possuir 7 espécies a mais que o PNB.

Comparando o presente estudo com outros similares (com ressalvas para o tipo de isca, período de amostragem e número de armadilhas), muitos também realizados em unidades de conservação de diferentes estados, percebe-se que os resultados são parecidos em alguns aspectos. No sul do país, no Paraná, em dois estudos realizados no Parque Estadual Mata dos Godoy, em mata e pastagem um coletou 4.069 indivíduos, 6 tribos e 40 espécies (Medrí \& Lopes, 2001) e o outro 4.687 indivíduos, 5 tribos e 27 espécies (Lopes et al., 2011). No Nordeste (Reserva Biológica Guaribas, na Paraíba, em Mata Atlântica e Tabuleiro Nordestino) foram coletados 3.533 indivíduos, 5 tribos e 29 espécies (Endres et al., 2007). No Norte, em Benjamin Constant, no Amazonas, foram coletados 6.792 indivíduos, 6 tribos e 63 espécies (Korasaki et al., 2012). No Centro-Oeste (Chapada dos Guimarães, Mato Grosso, em formações de savana, mata de Cerrado e Chaco) foram coletados 17.635 indivíduos, 6 tribos e 98 espécies (Daniel et al., 2014). Quando comparados ao Cerrado do Distrito Federal, os outros biomas apresentaram um maior número de indivíduos (principalmente na Chapada dos Guimarães), mas o número de tribos é semelhante, e o número de espécies foi largamente superado na Amazônia e em Mato Grosso, quando comparados ao PNB e PNCV separadamente.

A tribo Ateuchini, no presente estudo, foi representada principalmente pelos gêneros Genieridium e Uroxys. Genieridium cryptops foi a terceira espécie mais abundante no PNB, e no presente estudo como um todo. É distribuída pela América do Sul, incluindo Colômbia, Venezuela, Brasil, Bolívia, Paraguai e Argentina (Vazde-Mello, 2008), e comumente encontrada no Cerrado (Daniel et al., 2014).

A tribo Coprini foi representada principalmente pelos gêneros Canthidium e Dichotomius. Canthidium é o maior gênero em número de espécies na região Neotropical (Vaz-de-Mello et al., 2011). Canthidium decoratum foi a quarta espécie mais coletada no PNB. Almeida (2006) encontrou C. decoratum exclusivamente em 
ambientes abertos ou menos complexos, ao passo que no presente estudo, esta espécie foi coletada exclusivamente no PNB, que tem fitofisionomias mais fechadas que no $\mathrm{PNCV}$, onde a espécie não foi coletada.

Dichotomius é um gênero de ampla distribuição no ocidente, e é composto por 167 espécies descritas (Nunes \& Vaz-de-Mello, 2013), com hábito alimentar basicamente coprófago (Halffter \& Matthews, 1966). Dichotomius lycas foi a espécie mais abundante no PNCV, e é comumente encontrada no bioma Cerrado (Daniel et al., 2014).

Dentre os Delthochilini, Canthon foi o gênero mais abundante. É um gênero grande, com pelo menos 129 espécies descritas, endêmico das Américas, quase totalmente copro-necrófago (Vaz-de-Mello, 1999). Canthon aff. unicolor foi a segunda espécie mais coletada no PNCV. Daniel et al. (2014) registraram esta espécie exclusivamente em áreas florestais da Chapada dos Guimarães, Mato Grosso, ao passo que Almeida \& Louzada (2009) encontraram $C$. aff. unicolor majoritariamente em áreas de campo limpo, assim como neste estudo, onde esta espécie foi quase exclusivamente encontrada nesta fitofisionomia.

A tribo Phanaeini foi principalmente representada pelos gêneros Coprophanaeus, Oxysternon e Sulcophanaeus. Oxysternon é um gênero quase completamente restrito a América do Sul (Vaz-de-Mello, 1999). Oxysternon palemo foi a espécie mais abundante no PNB e no presente trabalho como um todo. É uma espécie associada a fezes humanas e bovinas, e é comumente encontrada em ambientes degradados, bem como em áreas urbanas em Brasília, Distrito Federal (Nunes et al., 2012).

Coprophanaeus é um gênero neotropical, ocorrendo em sua maioria na América do Sul, preferencialmente necrófago e de hábito noturno (Edmonds, 1972), e compreende ao menos 38 espécies descritas (Edmonds \& Zidek, 2010). Coprophanaeus spitzi foi a segunda espécie mais coletada no PNB e também a segunda mais abundante no presente estudo, sendo ela comumente encontrada no Cerrado (Edmonds \& Zidek, 2010). Em um trabalho comparando pastagens nativas e introduzidas, Almeida (2010) coletou indivíduos de C. spitzi com maior abundância em pastagens nativas. Por este motivo, esta espécie potencialmente pode ser utilizada como bioindicadora no PNB, sendo coletada quase exclusivamente nesta unidade, e com grande abundância. 
Sulcophanaeus menelas, a quarta espécie mais coletada no PNCV, é uma espécie que prefere ambientes mais abertos (Milhomem, 2003; Almeida, 2006), sendo coletada exclusivamente no PNCV, que possui fitofisionomias mais abertas que no PNB. A espécie também já foi encontrada no bioma Pampa, que possui formação aberta (Silva et al., 2012).

Apesar de no PNB ter sido coletado mais que o dobro do número de indivíduos comparado ao PNCV, se capturou menor número de espécies, e também de espécies exclusivas. Isto pode ter ocorrido devido ao menor tamanho da área do PNB e possivelmente pela grande urbanização que se encontra ao redor do parque, já que este fator afeta negativamente a comunidade de Scarabaeinae (Rodrigues \& Marchini, 1998; Korasaki et al., 2013). O grau de urbanização próximo ao habitat dos rola-bostas pode ter efeitos muito adversos, como a modificação do habitat ou redução da comunidade de mamíferos, cujas fezes são o seu principal recurso. Esses impactos podem ser mais negativos do que a fragmentação de habitat para os Scarabaeinae (Korasaki et al., 2013).

São relatadas 13 espécies de mamíferos de médio e grande porte no PNB e seis espécies no PNCV (Oliveira, 2010). A projeção do número de espécies de pequenos mamíferos não voadores no $\mathrm{PNB}$ é de 56 , ao passo que no $\mathrm{PNCV}$, esse número chega à 72 espécies (Silva, 2010). Mesmo com uma área maior e uma zona de amortecimento mais preservada, o PNCV possui menor número de espécies de mamíferos de médio e grande porte. Isto pode ocorrer pela predominância de áreas de campo nesta unidade, pois no estudo de Oliveira (2010), as duas unidades de conservação com predominância de áreas de campos apresentaram menor número de espécies de mamíferos. Ainda assim, no PNCV foram coletadas mais espécies de Scarabaeinae que no PNB, fato que pode ter ocorrido porque no PNB, a maioria das espécies de mamíferos relatadas são pertencentes à ordem Carnivora e como os rolabosta utilizam preferencialmente fezes de herbívoros e onívoros (Halffter \& Matthews, 1966; Dormont et al., 2004), é possível que os recursos (fezes) no PNB não sejam tão atrativos como no PNCV, favorecendo o estabelecimento de um maior número de espécies de besouros.

Outro fator que pode explicar a riqueza encontrada nas duas unidades de conservação é a heterogeneidade do habitat. Áreas de Cerrado com alta heterogeneidade têm uma relação negativa com a riqueza de rola-bostas, possivelmente pelo aumento da heterogeneidade não refletir no aumento da 
disponibilidade de recursos (Da Silva et al., 2010). No presente estudo, o PNB apresenta maior heterogeneidade de habitat que o PNCV (pelo módulo estar inserido principalmente em Cerrado sensu stricto, ao passo que no PNCV o módulo se encontra predominantemente em Campo limpo), podendo esta ser uma explicação para a menor riqueza encontrada neste local. No Cerrado, áreas mais abertas costumam possuir maior riqueza e abundância de Scarabaeinae (Milhomem, 2003).

A maior riqueza, mesmo não sendo significativa, encontrada no PNCV pode estar relacionada às fitofisionomias mais abertas, preferencialmente utilizadas pelos Scarabaeinae, ao passo que a maior abundância encontrada no PNB pode estar relacionada ao maior número de mamíferos de médio e grande porte. Mesmo que predominem carnívoros no PNB, a riqueza de mamíferos ainda é maior que no PNCV.

Outro aspecto que pode explicar a maior abundância de Scarabaeinae encontrada no PNB é que em ambientes mais degradados ou antropizados (próximos ao lixão da Estrutural, ocorrência de caça clandestina, presença de animais domésticos) as espécies de rola-bosta generalistas se estabelecem mais facilmente, utilizando um nicho pouco utilizado por outras espécies, e podem dominar o ambiente como ocorreu com O. palemo.

No PNB houve dominância da espécie O. palemo, que representou $35 \%$ do total coletado, ao passo que no PNCV a abundância das espécies foi mais uniforme, sendo que D. lycas, a espécie mais coletada, representou $15,1 \%$ do total. Sabe-se que em ambientes mais antropizados ocorrem menor número de espécies, com maior dominância de algumas delas, e em ambientes mais preservados, ocorrem maior número de espécies, distribuídas de forma mais uniforme (Nichols et al., 2007; Korasaki et al., 2013).

Ao compararmos a diversidade de Scarabaeinae no Cerrado, o presente estudo apresenta índices de valor intermediário, H' de 2,055 no PNB e H' de 2,898 no PNCV, ao passo que outro estudo no Cerrado de Mato Grosso do Sul encontrou índices maiores $(4,68 ; 4,33$ e 4,95) (Koller et al., 2007) e um terceiro no Cerrado do Distrito Federal apresentou índices de valor 1,233; 1,261 e 0,827 (Milhomem, 2003).

A maioria das espécies coletadas foi pertencente à guilda funcional dos paracoprídeos (escavadores), corroborando resultados de outros estudos (Louzada \& Lopes, 1997; Milhomem, 2003). Os paracoprídeos cavam túneis mais profundos abaixo do recurso, carregando fezes e acidentalmente, sementes, ao passo que 
telecoprídeos rolam as sementes que estão na massa fecal (também de maneira acidental) que transportam para longe do recurso, e posteriormente as enterram (Vulinec, 2002). Este padrão talvez possa ser explicado devido aos paracoprídeos, principalmente os de maior tamanho, serem mais eficientes na degradação de uma fonte de excremento, sendo esta guilda funcional a mais importante tanto na degradação de fezes, como na dispersão secundária de sementes (Slade et al., 2007), os tornando competitivamente eficientes. Louzada \& Lopes (1997) acreditam que por este ser um padrão da região Neotropical, as coletas em diferentes áreas funcionam como subamostras, refletindo a proporção da comunidade encontrada nesta região. Além disso, solos compactados, normalmente ricos em partículas de argila, tendem a ser desafiadores para os paracoprídeos cavarem seus túneis profundos, podendo favorecer os telecoprídeos e principalmente os endocoprídeos (Silva et al., 2015).

No presente estudo, a distribuição dos indivíduos corrobora a encontrada na literatura (Silva et al., 2012), onde as espécies paracoprídeas (escavadoras) preferem áreas mais fechadas, e as telecoprídeas (roladoras) preferem áreas mais abertas. Podese ver que os Deltochilini, tribo predominantemente de espécies telecoprídeas, foram mais coletados no PNCV (onde o módulo possui a maioria das parcelas em áreas de Campo limpo), ao passo que os Phanaeini, tribo predominantemente de espécies paracoprídeas, foram mais coletados no PNB (onde o módulo possui a maioria das parcelas em áreas de Cerrado sensu stricto).

O tamanho dos escarabeíneos é um fator que pode ajudar a inferir como é a comunidade de mamíferos de uma área, ou quão bem preservada essa área é. Diversos trabalhos demonstraram que normalmente, rola-bostas maiores são mais afetados por impactos negativos do que os pequenos (Scheffler, 2005; Larsen et al., 2008; Lee et al., 2009; Andrade et al., 2014). Rola-bostas menores são mais abundantes em áreas desmatadas (Vulinec, 2002), provavelmente sendo mais resistentes que os besouros maiores. Nas duas unidades de conservação avaliadas no presente trabalho, foi encontrado maior número de espécies consideradas pequenas $(<10 \mathrm{~mm})$, porém nas duas unidades as espécies maiores foram mais abundantes. Esta expressiva abundância de espécies de grande tamanho corporal $(>10 \mathrm{~mm})$, que normalmente são muito suscetíveis à impactos, pode indicar que ambas unidades de conservação estão preservadas.

Os insetos são animais de pequeno porte, e de modo geral, tem um ciclo de vida curto e são suscetíveis a mudanças no seu ecossistema, os tornando bons 
organismos bioindicadores de qualidade do meio ambiente (Brown Jr., 1997). Eurysternus caribaeus pode ser classificado como uma espécie bioindicadora, pois ela possui baixa tolerância para ambientes alterados (Granados et al., 2010). Vulinec (2002) encontrou resultados onde telecoprídeos e phaneíneos foram afetados pela degradação do habitat na Amazônia, ao passo que Dichotomius apresentou maior resistência a alterações. No presente estudo, Phanaeini representou a maior parte da coleta, principalmente no PNB, ao passo que Deltochilini (telecoprídeos) foi mais coletada no PNCV, bem como Dichotomius, que foi quase exclusivamente coletado no PNCV. Mais estudos nas duas unidades de conservação seriam interessantes, avaliando as comunidades de Phanaeini no PNB e de Delthochilini no PNCV, a fim de verificar se esses grupos são bioindicadores viáveis nessas duas unidades de conservação.

$\mathrm{O}$ PNB e o PNCV apresentaram valores intermediários do índice de diversidade de Shannon-Wiener, quando comparados a outras áreas de Cerrado. As duas unidades de conservação suportam a comunidade de Scarabaeinae presente em seus limites, fator que indica que a comunidade de mamíferos também deve estar protegida. Mesmo com as desvantagens que o PNB enfrenta (menor área, matriz urbana, proximidade ao aterro de lixo, entrada de pessoas não autorizadas e animais domésticos) a comunidade de rola-bostas na região é comparável com outras no Cerrado. As duas unidades de conservação são de extrema importância e devem continuar sendo preservadas.

\section{5 - CONCLUSÃO}

No presente trabalho, não foi encontrada diferença estatística na riqueza de espécies de Scarabaeinae entre as unidades de conservação avaliadas (PNB e PNCV), porém houve diferença estatística na abundância dos indivíduos.

As comunidades de Scarabaeinae são distintas entre as duas Unidades de Conservação, de acordo com o Índice de Sorensen.

As duas unidades de conservação possuem espécies pertencentes às três guildas funcionais em seus limites, sendo as paracoprídeas as mais abundantes, seguidas das telecoprídeas e endocoprídeas. 
Um maior número de espécies pequenas $(<10 \mathrm{~mm})$ foi coletado nas duas unidades de conservação, porém as espécies grandes (>10 mm) foram mais abundantes.

\section{6 - REFERÊNCIAS BIBLIOGRÁFICAS}

AGOGLITTA, R.; ROSSINI, M. \& ZUNINO, M. El monitereo del cambio climático y los coleópteros Scarabaeoidea coprófagos (Coleoptra: Scarabaeoidea): propuesta preliminar. Boletín de la Sociedad Entomológica Aragonesa. 46: 181-187. 2010.

AL-HOUTY, W. \& AL-MUSALAM, F. Dung preference of the dung beetle Scarabaeus cristatus Fab (Coleoptera-Scarabaeidae) form Kuwait. Journal of Arid Environments. 35: 511-516. 1997.

ALMEIDA, S. S. P. Diversidade de Scarabaeidae s. str. detritívoros (Coleoptera) em diferentes fitofisionomias da Chapada dos Perdizes, Carrancas-MG. Dissertação, Universidade Federal de Lavras. 2006.

ALMEIDA, S. S. P. Substituição de pastagem nativa por Braquiária: impactos na estrutura e funções ecológicas da comunidade de escarabeíneos (Coleoptera). Tese, Universidade Federal de Viçosa. 2010.

ALMEIDA, S. S. P. \& LOUZADA, J. N. C. Estrutura da Comunidade de Scarabaeinae (Scarabaeide: Coleoptera) em Fitofisionomias do Cerrado e sua Importância para a Conservação. Neotropical Entomology, 38(1): 32-43. 2009.

ALVES, F. R.; FRANÇA, F. M.; MACEDO, R. S.; NICOLAI, L. H. T.; BRAGA, R. F. \& LOUZADA, J. N. C. Serviços ambientais prestados por Scarabaeinae (Coleoptera, Scarabaeidae) em área de Floresta Amazônica, Monte Dourado - PA. Anais do IX Congresso de Ecologia do Brasil. 2009.

AMÉZQUITA, S. \& FAVILA, M. E. Removal Rates of Native and Exotic Dung by Dung Beetles (Scarabaeidae: Scarabaeinae) in Fragmented Tropical Rain Forest. Environmental Entomology. 39(2): 328-336, 2010. 
ANDERSEN, A. N.; HOFFMANN, B. D.; MUlLER, W. J. \& GRIFFITHS, A. D. Using ants as bioindicators in land management: simplifying assessment of ant community responses. Journal of Applied Ecology. 38: 8-17. 2002.

ANDRADE, R. B.; BARLOW, J.; LOUZADA, J.; VAZ-DE-MELLO, F. Z.; SILVEIRA, J. M \& COCHRANE, M. A. Tropical forest fires and biodiversity: dung beetle community and biomass responses in a northern Brazilian Amazon forest. Insect Conservation. 18: 1097-1104. 2014.

ANDRESEN, E. \& LAURANCE, S. G. W. Possible indirect effects of mammal hunting on dung beetle assemblages in Panama. Biotropica. 39(1): 141-146. 2007.

ARAÚJO, L. S.; PIMENTA, L.; BATISTA, T. A. \& SOUZA-SILVA, H. Por que a riqueza e abundância de besouros coprófagos (Coleoptera: Scarabaeidae) aumenta quanto menos antropizado o habitat? Anais do IX Congresso de Ecologia do Brasil. São Lourenço, MG. 2009.

BEULCHLE, R.; GRECCHI R. C.; SHIMABUKURO, Y. E.; SELINGER, R.; EVA, H. D.; SANO, E. \& ACHARD, F. Land cover changes in the Brazilan Cerrado and Caatinga biomes from 1990 to 2010 based on systemic remote sensing sampling approach. Applied Geography. 58:116-127. 2015.

BROWN JR., K. S. Diversity, disturbance, and sustainable use of Neotropical forests: insects as indicators for conservation monitoring. Journal of Insect Conservation. 1: 25-42. 1997.

CAMBEFORT, Y. \& HANSKI, I. Dung beetle population biology. In: HANSKI, I.; CAMBEFORT, Y. (Eds.), Dung Beetle Ecology. Princeton University Press, Princeton, NJ, pp. 36-50. 1991.

COLWELL, R. K. EstimateS, Version 9.1: Statistical estimation of species richness and shared species from samples $<$ purl.oclc.org/estimates>. 2013. 
DANIEL, G. M.; NUNES, L. G. O. A. \& VAZ-DE-MELlO, F. Z. Species composition and functional guilds of dung beetles (Insecta: Coleoptera: Scarabaeidae: Scarabaeinae) in different vegetation types in the brazilian Shield-Chacoan depression border. Annales de la Société Entomologique de France (N.S.). 1-8. 2014.

DA SILVA, P. G. \& DI MARE, R. A. Escarabeíneos copro-necrófagos (Coleoptera, Scarabaeidae, Scarabaeinae) de fragmentos de Mata Atlântica em Silveira Martins, Rio Grande do Sul, Brasil. Iheringia, Série Zoologia. 102(2): 197-205. 2012.

DA SILVA, P. G.; VAZ-DE-MELlO, F. Z. \& DI MARE, R. A. Attractiveness of different bait to the Scarabaeinae (Coleoptera: Scarabaeidae) in forest fragments in extreme Southern Brazil. Zoological Studies. 51(4): 429-441. 2012.

DA SILVA, R. J.; DINIZ, S. \& VAZ-DE-MELLO, F. Z. Heterogeneidade do habitat, riqueza e estrutura da assembléia de besouro rola-bostas (Scarabaeidae: Scarabaeinae) em áreas de Cerrado na Chapada dos Parecis, MT. Neotropical Entomology. 39(6): 934-940. 2010.

DORMONT, L.; EPINAT, G. \& LUMARET, J. P. Trophic preferences mediated by olfactory cues in dung beetles colonizing cattle and horse dung. Environmental Entomology. 33(2): 370-377. 2004.

EDMONDS, W. D. Comparative skeletal morphology, systematics and evolution of the Phanaeini dung beetle (Coleoptera: Scarabaeidae). The University of Kansas Science Bulletin. 49(11): 731-874. 1972.

EDMONDS, W. D. \& ZIDEK, J. A taxonomic review of the neotropical genus Coprophanaeus Olsoufieff, 1924 (Coleoptera: Scarabaeidae: Scarabaeinae). Insecta Mundi. 129: 1-111. 2010.

ENDRES, E. E.; CREÃO-DUARTE, A. J. \& HERNÁNDEZ, M. I. M. Diversidade de Scarabaeinae s. str. (Coleoptera) da Reserva Biológica Guaribas, Mamanguape, Paraíba, Brasil: uma comparação entre Mata Atlântica e Tabuleiro Nordestino. Revista Brasileira de Entomologia. 51(1): 67-71. 2007. 
ESCOBAR, F.; HALFFTER, G.; SOLÍS, A.; HALFFTER, V. \& NAVARRETE, V. Temporal shifts in dung beetle community structure within a protected area of tropical wet forest: a 35-year study and its implications for long-term conservation. Journal of Applied Ecology. 45: 1584-1592. 2008.

FAVILA, M. E. \& HALFFTER, G. The use of indicator groups for measuring biodiversity as related to community structure and function. Acta Zoologica Mexicana. 72: 1-25. 1997.

FILGUEIRAS, B. K. C.; LiBERAL, C. N.; AGUIAR, C. D. M.; HERNÁNDEZ, M. I. M. \& IANNUZZI, L. Attractivity of omnivore, carnivore and herbivore mammalian dung to Scarabaeinae (Coleoptera, Scarabaeidae) in a tropical Atlantic rainforest remnant. Revista Brasileira de Entomologia. 53(3): 422-427. 2009.

FURLEY, P. A. The Nature and diversity of neotropical savanna vegetation with particular reference to the Brazilian Cerrados. Global Ecology and Biogeography. 8: 223-241. 1998.

GARDNER, T. A.; HERNÁNDEZ, M. I. M.; BARLOW, J. \& PERES, C. A. Understanding the biodiversity consequences of habitat change: the value of secondary and plantation forests for neotropical dung beetles. Journal of Applied Ecology. 1-11. 2008.

GRANADOS, J. M.; KOHLMANN, B. \& RUSSO, R. Escrabájos del estiércol como bioindicadores del impacto ambiental causado por cultivos en la región Atlântica de Costa Rica. Tierra Tropical. 6(2): 181-189. 2010.

HALFFTER, G. \& MATTHEWS, E. G. The natural history of dung beetles of the subfamily Scarabaeinae. Folia Entomologica Mexicana. 12-14: 1-312. 1966.

HALFFTER, G. Historical end ecological factors determining the geographical distribution of beetles (Coleoptera: Scarabaidae: Scarabaeinae). Folia Entomológica Mexicana. 82: 195-238, 1991. 
HANSKI, I. Nutritional Ecology of Dung and Carrion Feeding Insects. In: Slanky, Jr., F. \& Rodriguez, J.G. (Eds), Nutritional Ecology of Insects, Mites and Spiders, pp. 837-884. New York: Wiley. 902 pp. 1987.

KLINK, C. A. \& MACHADO, R. B. Conservation of the Brazilian Cerrado. Conservation Biology. 19(3): 707-713. 2005.

KOLLER, W. W.; GOMES, A.; RODRIGUES, S. R. \& GOIOZO, P. F. I. Scarabaeidae e Aphodiidae coprófagos em pastagens cultivadas em area do Cerrado sul-mato-grossense. Revista Brasileira de Zoociências. 9(1): 81-93. 2007.

KORASAKI, V.; VAZ-DE-MELLO, F. Z.; BRAGA, R. F.; ZANETTI, R \& LOUZADA, J. Taxocenose de Scarabaeinae (Coleoptera:Scarabaeidae) em Banjamin Constant, AM. Acta Amazonica. 42(3): 423-432. 2012.

KORASAKI, V.; LOPES, J.; BROWN, G. G. \& LOUZADA, J. Using dung beetles to evaluate the effects of urbanization on Atlantic Forest biodiversity. Insect Science. 20: 393-406. 2013.

INSTITUTO CHICO MENDES DE CONSERVAÇÃO DA BIODIVERSIDADE (ICMBio). Plano de manejo do Parque Nacional de Brasília. Disponível em: $<$ http://www.icmbio.org.br/portal/imagens/stories/imgsunidadeconservação/PARNA \%20Brasilia.pdf> Acesso em: 1 de agosto de 2016.

INSTITUTO CHICO MENDES DE CONSERVAÇÃO DA BIODIVERSIDADE (ICMBio). Plano de manejo do Parque Nacional da Chapada dos Veadeiros. Disponível em: $<\underline{\text { http://www.icmbio.gov.br/portal/images/stories/imgs-unidades- }}$ coservacao/pm_chapada dos_veadeiros 1.pdf> Acesso em: 1 de agosto de 2016.

LARSEN, T. H.; LOPERA, A. \& FORSYTH, A. Understanding trait-dependent community disassembly: dung beetles, density functions and forest fragmentation. Conservation Biology. 22(5): 1288-1298. 2008. 
LEE, J. S.H.; LEE, I. Q. W.; LIM, S. L. H.; HUIJBREGTS, J \& SODHI, N. S. Changes in dung beetle communities along a gradiente of tropical forest disturbance in South-East Asia. Journal of Tropical Ecology. 25: 677-680. 2009.

LIMA, P. C. A. \& FRANCO, J. L. A. As RPPNS como estratégias para a conservação da biodiversidade: o caso da Chapada dos Veadeiros. Sociedade \& Natureza. 26(1): 113-125. 2014.

LOPES, J.; KORASAKI, V.; CATELLI, L. L.; MARÇAL, V. V. M. \& NUNES, M. P. B. P. A comparison of dung beetle assemblage structure (Coleoptera: Scarabaidae: Scarabaeinae) between an Atlantic forest fragment and adjacent abandoned pasture in Paraná, Brasil. Sociedade Brasileira de Zoologia. 28(1): 72-79. 2011.

LOUZADA, J. N. C. \& LOPES, F. S. A comunidade de Scarabaidae copronecrófagos (Coleoptera) de um fragmento de Mata Atlântica. Revista Brasileira de Entomologia. 41(1): 117-121. 1997.

LUÇARDO, M.; OLIVEIRA, C. M. \& FRIZZAS, M. R. Scarabaeoidea (Insecta: Coleoptera) no Cerrado brasileiro: estado atual de conhecimento. Ciência Rural. 44(4): 652-659, 2014.

LUNZ, A. M.; VAZ-DE-MELLO, F. Z. \& MONTEIRO, O. M. Pelidnota fillipinae Soula, 2009 (Copeoptera: Melolonthidae: Rutelinae): um novo desfolhador de acaciaaustraliana, Racosperma mangium (Wild.) Pedley (Fabaceae), na Amazônia Oriental, Brasil. Acta Amazonica. 41(3): 439-442. 2011.

MAZORRA, M. A.; SAN JOSE, J. J.; MONTES, R.; GACIA MIRAGAYA, J. \& HARIDASAN, M. Aluminium concentration in the biomass of native species of the Morichals (swamp palm community) at the Orinoco Llanos, Venezuela. Plant and Soil. 102: 275-277. 1987.

MCGEOCH, M. A.; VAN RENSBURG, B. J. \& BOTES, A. The verification and application of bioindicators: a case study of dung beetles in a savannah ecosystem. Journal of Applied Ecology. 39: 661-672. 2002. 
MEDRÍ, I. M. \& LOPES, J. Scarabaeidae (Coleoptera) do Parque Estadual Mata dos Godoy e de área de pastagem, no norte do Paraná, Brasil. Revista Brasileira de Zoologia. 18(1): 135-141. 2001.

MILHOMEM, M. S. A fauna de Scarabaeidae sensu stricto (Coleoptera: Scarabaeoidea) do Cerrado de Brasília, DF: variação anual, efeito do fogo e cobertura vegetal. Tese, Universidade de Brasília, 2003.

MINISTÉRIO DO MEIO AMBIENTE. MMA. Catálogo taxonômico da fauna do Brasil. 2016a. Disponível em <http://www.mma.gov.br/catalogo-fauna>. Acesso em: 24 de maio de 2016.

MINISTÉRIO DO MEIO AMBIENTE. MMA. Unidades de Conservação, o que são? 2016b. Disponível em $<$ http://www.mma.gov.br/areas-protegidas/unidades-deconservacao/o-que-sao>. Acesso em: 27 abril de 2016.

MYERS, N.; MITTERMEIER, R. A.; MITTERMEIER, C. G.; FONSECA, G. A. B. \& KENT, J. Biodiversity hotspots for conservation priorities. Nature. 403: 853-858. 2000.

NICHOLS, E.; LARSEN, T.; SPECTOR, S.; DAVIS, A. L.; ESCOBAR, F.; FAVILA, M. \& VULINEC, K. Global dung beetle response to tropical forest modification and fragmentation: A quantitative literature review and meta-analyses. Biological Conservation. 137: 1-19. 2007.

NICHOLS, E.; SPECTOR, S.; LOUZADA, J.; LARSEN, T.; AMEZQUITA, S. \& FAVILA, M. E. Ecological functions and ecosystem services of Scarabaeinae dung beetles. Biological Conservation. 141: 1461-1474. 2008.

NUNES, R. V.; FRIZZAS, M. R. \& VAZ-DE-MELLO, F. Z. Scarabaeinae (Coleoptera: Scarabaeidae) of a rupestrian field at Cafuringa, Distrito Federal, Brazil: commented list of species. Biota Neotropical. 12(4): 125-129. 2012. 
NUNES, R. V. \& VAZ-DE-MELLO, F. Z. New brachypterous species of Dichotomius Hope, with taxonomic notes in the subgenus Luederwaldtinia Martínez (Coleoptera: Scarabaeidae: Scarabaeinae). Zootaxa. 3609(4): 411-420. 2013.

OLIVEIRA, I. M. Riqueza, abundância de espécies e uso de habitat por mamíferos de médio e grande porte em cinco unidades de conservação no Cerrado. Dissertação, Universidade de Brasília. 2010.

OLIVEIRA, V. H. F.; SOUZA, J. G. M.; VAZ-DE-MELLO, F. Z.; NEVES, F. S \& FAGUNDES, M. Variação na fauna de besouros rola-bosta (Coleoptera: Scarabainae) entre habitats de cerrado, mata seca e mata ciliar em uma região de transição Cerrado - Caatinga no norte de Minas Gerais. MG. Biota. 4(4): 4-16. 2011.

PESSOA, K. K. T. Besouros Escarabeíneos (Insecta: Coleoptera: Scarabaeidae) Atraídos por Víscera Bovina em um Remanescente de Mata Atlântica de Pernambuco, Durante a Estação Seca. Monografia, Universidade Federal Rural de Pernambuco. 2010.

PONCE-SANTIZO, G.; ANDRESEN, E.; CANO, E. \& CUARÓN, A. D. Dispersión primaria de semillas por primates y dispersión secundaria por escarabajos coprófagos en Tikal, Guatemala. Biotropica. 38(3): 390-397. 2006.

Programa de Pesquisa em Biodiversidade (PPBio). 2012. Disponível em $<$ https://ppbio.inpa.gov.br $>$. Acesso em: 1 de agosto de 2016.

R DEVELOPMENT CORE TEAM. R: a language and environment for statistical computing. Vienna: R Foundation for Statistical Computing, 2016. <https://www.rproject.org/>.

RAINIO, J \& NIEMELA, J. Ground beetles (Coleoptera: Carabidae) as bioindicators. Biodiversity and Conservation. 12: 487-506. 2003.

RATTER, J. A.; RIBEIRO, J. F. \& BRIDGEWATER, S. The Brazilian Cerrado Vegetation and Threats to its Biodiversity. Annals of Botany. 80: 223-230. 1997. 
RIDSDILL-SMITH, T. J., HAYLES, L. \& PALMER, M. J. Competition between the bush fly and a dung beetle in dung of differing characteristics. Entomologia Experimentalis et Applicata. 41: 83-90. 1986.

RODRIGUES, S. R. \& MARCHINI, L. C. Besouros coprófagos (Coleoptera: Scarabaeidae) coletados em Piracicaba, SP. Scientia Agricola. 55(1): 53-58. 1998.

RONQUI, D. C. \& LOPES, J. Composição e diversidade de Scarabaeioidea (Coleoptera) atraídos por armadilha de luz em área rural no norte do Paraná, Brasil. Iheringia. Série Zoologia. 96 (1): 103-108. 2006.

SANCHEZ, T. V. Convergência ecológica de comunidades de Scarabainae em sistemas nativos e pastagens da América do Sul. Dissertação, Universidade Federal de Lavras. 2011.

SANO, E.; ROSA, R.; BRITO, J. L. S. \& FERREIRA, L. G. Land cover mapping of the tropical savana region in Brazil. Environmental Monitorament Assess. 166: 113124.2009.

SANTOS, V. \& ÁVILA, C, J. Aspéctos biológicos e comportamentais de Liogenys suturalis Blanchard (Coleoptera:Melolonthidae) no Mato Grosso do Sul. Neotropical Entomology. 38(6): 734-740. 2009.

SCHEFFLER, P. Y. Dung beetle (Coleoptera: Scarabaeinae) diversity and community structure across three disturbance regimes in eastern Amazonia. Journal of Tropical Ecology. 21: 9-19. 2005.

SILVA, P. G.; AUDINO, L. D.; NOGUEIRA, J. M.; MORAES, L. P. \& VAZ-DEMELLO, F. Z. Escarabeíneos (Coleoptera: Scarabaeidae: Scarabaeinae) de uma área de campo nativo no bioma Pampa, Rio Grande do Sul, Brasil. Biota Neoptropical. 12(3): 1-8. 2012. 
SILVA, R. J.; RIBEIRO, H. V.; SOUZA, M. F. \& VAZ-DE-MELLO, F. Z. Influência da granulometria do solo na estrutura de guildas funcionais de besouros rola-bostas (Coleoptera: Scarabaeidae: Scarabaeinae) em florestas semideciduais no estado do Mato Grosso, Brasil. Bioscience Journal. 31(2): 601-612. 2015.

SILVA, R. R. Padrões de riqueza e distribuição de espécies de pequenos mamíferos terrestres do Cerrado: uma ferramenta para modelagem e avaliação do sistema de unidades de conservação. Tese, Universidade de Brasília. 2010.

SLADE, E. M.; MANN, D. J.; VILLANUEVA, J. F \& LEWIS, O. Experimental evidence for the effects of dung beelte functional group richness and composition on ecossystem function in a tropical forest. Journal of Animal Ecology. 76: 1094-1104. 2007.

VAZ-DE-MELLO, F. Z. Scarabaidae s. str. (Coleoptera: Scarabaeoidea) de um fragmento de Floresta Amazônica do estado do Acre, Brasil. 1. Taxocenose. Anais Sociedade Entomológica do Brasil. 28(3): 447-453. 1999.

VAZ-DE-MELLO, F. Z. Estado Atual dos Scarabaeoidea s.str. (Coleoptera: Scarabaeoidea) do Brasil. Monografías Tercer Milenio, 1: 183-195. 2000.

VAZ-DE-MELLO, F. Z. Synopsis of the new subtribe Scatimina (Coleoptera: Scarabaeidae: Scarabaeinae: Ateuchini), with descriptions of twelve new genera and review of Genieridium, new genus. Zootaxa. 1955: 1-75. 2008.

VAZ-DE-MELLO, F. Z.; EDMONDS, W. D.; OCAMPO, F. C. \& SHOOLMEESTERS, P. A multilingual key to the genera and subgenera of the subfamily Scarabaeinae of the New World (Coleoptera: Scarabaeidae). Zootaxa. 2854: 1-73. 2011.

VAZ-DE-MELlO, F. Z.; SILVA, R. L. R.; NUNES, L. G. O. A. \& CORRÊA, P. R. O. Os Besouros Rola-Bosta (Insecta: Coleoptera: Scarabaeidae: Scarabaeinae) da Fazenda São Nicolau. Descobrindo a Amazônia Meridional: Biodiversidade da Fazenda São Nicolau, cap. 4. 77-102. 2011. 
VULINEC, K. Dung beetle communities and seed dispersal in primary forest and disturbed land in Amazonia. Biotropica. 34(2): 297-309. 2002.

WINK, C.; GUEDES, J. V. C.; FAGUNDES, C. K. \& ROVEDDER, A. P. Insetos edáficos como indicadores de qualidade ambiental. Revista de Ciências Agroveterinárias. 4 (1): 60-61. 2005.

ZUNINO, M. Food Relocation Behaviour: a multivalente strategy for Coleoptera. Advances in Coleopterology. 297-314, 1991. 